\title{
Constant mean curvature surfaces in 3-dimensional Thurston geometries
}

\author{
Isabel Fernández and Pablo Mira
}

\begin{abstract}
This is a survey on the global theory of constant mean curvature surfaces in Riemannian homogeneous 3-manifolds. These ambient 3-manifolds include the eight canonical Thurston 3-dimensional geometries, i.e. $\mathbb{R}^{3}, \mathbb{H}^{3}, \mathbb{S}^{3}, \mathbb{H}^{2} \times \mathbb{R}, \mathbb{S}^{2} \times \mathbb{R}$, the Heisenberg space $\mathrm{Nil}_{3}$, the universal cover of $\mathrm{PSL}_{2}(\mathbb{R})$ and the Lie group $\mathrm{Sol}_{3}$. We will focus on the problems of classifying compact CMC surfaces and entire CMC graphs in these spaces. A collection of important open problems of the theory is also presented.
\end{abstract}

Keywords. Constant mean curvature surfaces, homogeneous spaces, Thurston geometries, harmonic maps, minimal surfaces, entire graphs.

\section{Introduction}

Constant mean curvature (CMC) surfaces appear as critical points of a natural geometric variational problem: to minimize surface area with or without a volume constraint (the unconstrained case corresponds to zero mean curvature, i.e. to minimal surfaces). A fundamental problem of this discipline is the geometric study and classification of CMC surfaces under global hypotheses like compactness, completeness, properness or embeddedness. The study of this problem for CMC surfaces in the model spaces $\mathbb{R}^{3}, \mathbb{S}^{3}$ and $\mathbb{H}^{3}$ has produced a very rich theory, in which geometric arguments interact with complex analysis, harmonic maps, integrable systems, maximum principles, elliptic PDEs, geometric measure theory and so on.

One of the most remarkable achievements of this field in the last decade has been the extension of this classical theory to the case of CMC surfaces in simply connected homogeneous 3-dimensional ambient spaces. Apart from $\mathbb{R}^{3}, \mathbb{S}^{3}$ and $\mathbb{H}^{3}$, these spaces are the remaining five Thurston 3-dimensional geometries (i.e. $\mathbb{H}^{2} \times \mathbb{R}, \mathbb{S}^{2} \times \mathbb{R}$, the Heisenberg group $\mathrm{Nil}_{3}$, the universal covering of $\mathrm{PSL}_{2}(\mathbb{R})$ and the Lie group $\left.\mathrm{Sol}_{3}\right)$, together with 3dimensional Berger spheres and some other Lie groups with left-invariant metrics (see Section 2).

It must be said here that there is an important number of contributions regarding CMC surfaces in general Riemannian 3-manifolds (not even homogeneous), many of which deal for instance with isoperimetric questions or with geometric consequences derived from the stability operator associated to the second variation of the surface. The achievement in the case of homogeneous ambient 3-spaces has been the construction of a very rich global theory of CMC surfaces, analogous to the case of $\mathbb{R}^{3}, \mathbb{S}^{3}$ and $\mathbb{H}^{3}$, with an emphasis on the geometric classification (up to ambient isometries) of properly immersed or properly 
embedded CMC surfaces. The fact that the ambient space is homogeneous, i.e. it has the same local geometry at all points, makes this problem extremely natural.

Our aim here is to present a survey on some fundamental aspects of the global theory of CMC surfaces in homogeneous 3-manifolds. We do not plan, however, to give a systematic account of all important results of this already broad theory, but to discuss some specific problems at the core of it. Hence, there will be many important results omitted, and we apologize in advance for that.

In order to explain the problems we shall be dealing with, let us distinguish between compact and non-compact CMC surfaces in these spaces.

In the case of compact CMC surfaces, three fundamental problems are the Alexandrov problem (i.e. to classify compact embedded CMC surfaces), the Hopf problem (i.e. to classify CMC spheres), and the isoperimetric problem (recall that isoperimetric regions on a Riemannian 3-manifold are bounded by compact embedded CMC surfaces, but the converse is not always true). By classical results, round spheres constitute the solution to each of these three problems in the case of CMC surfaces in $\mathbb{R}^{3}$. One of our main objectives will be to explain what is known (and what is not known) for these problems in the broader context of CMC surfaces in homogeneous 3-manifolds.

In the case of non-compact CMC surfaces, one of the basic problems is to study the properly embedded CMC surfaces of finite topology. A classical result in that direction is given by Bernstein's theorem: planes are the only entire minimal graphs in $\mathbb{R}^{3}$. As in all Thurston 3-dimensional geometries there is a natural notion of entire graph, it is an important problem of the discipline to solve the Bernstein problem for CMC graphs, i.e. to classify all entire CMC graphs in these 3-dimensional ambient spaces. This will be our other main objective.

The theory of CMC surfaces in Thurston 3-dimensional geometries started to develop as a consistent unified theory after some pioneer works by Harold Rosenberg, jointly with William H. Meeks MeRo1, MeRo2, Ros for the case of minimal surfaces in product spaces, and jointly with Uwe Abresch AbRo1, AbRo2 for the case of CMC surfaces in homogeneous spaces with a 4-dimensional isometry group.

On one hand, Meeks and Rosenberg established many results on complete minimal surfaces in $M^{2} \times \mathbb{R}$, what has guided a large number of subsequent works in the field. A recent major contribution in this sense is the Collin-Rosenberg theorem [CoRo] on the existence of harmonic diffeomorphims from $\mathbb{C}$ onto the hyperbolic plane $\mathbb{H}^{2}$, obtained by constructing an entire minimal graph of parabolic conformal type in $\mathbb{H}^{2} \times \mathbb{R}$.

On the other hand, Abresch and Rosenberg discovered a holomorphic quadratic differential for CMC surfaces in these homogeneous spaces with 4-dimensional isometry group (the $\mathbb{E}^{3}(\kappa, \tau)$ spaces), and solved the Hopf problem for them. The general integrability theory of CMC surfaces in the homogeneous $\mathbb{E}^{3}(\kappa, \tau)$ spaces was then established by B. Daniel Dan1. The discovery by the authors of a harmonic Gauss map into $\mathbb{H}^{2}$ for $H=1 / 2$ surfaces in $\mathbb{H}^{2} \times \mathbb{R}$ turned into a series of papers by Daniel, Fernández, Hauswirth, Mira, Rosenberg, Spruck [FeMi1, Dan2, FeMi2, HRS, DaHa] in which the Bernstein problem for CMC graphs of critical mean curvature (including minimal graphs in Heisenberg space $\mathrm{Nil}_{3}$, see Section 6) was solved. Very recently, the Hopf and Alexandrov problems for CMC surfaces have been solved by Daniel-Mira and Meeks DaMi, Mee] in the remaining Thurston 3-dimensional geometry: the Lie group $\mathrm{Sol}_{3}$, whose isometry group is only 3 -dimensional.

We have organized this exposition as follows. In Section 2 we will introduce the 3 -dimensional homogeneous ambient spaces. In Section 3 we will present the basic integrability equations by Daniel for CMC surfaces in the homogeneous spaces $\mathbb{E}^{3}(\kappa, \tau)$, 
together with the holomorphic Abresch-Rosenberg differential, and with some basic definitions on stability of CMC surfaces. In Section 4 we will discuss the Hopf, Alexandrov and isoperimetric problems in the homogeneous spaces $\mathbb{E}^{3}(\kappa, \tau)$. Section 5 will be devoted to solving the Hopf and Alexandrov problems in the eighth Thurston geometry, i.e. the Lie group $\mathrm{Sol}_{3}$. In Section 6 we will present the solution to the Bernstein problem for entire graphs of critical CMC in the homogeneous $\mathbb{E}^{3}(\kappa, \tau)$ spaces. Finally, in Section 7 we shall expose the Collin-Rosenberg theorem on parabolic entire minimal graphs in

$\mathbb{H}^{2} \times \mathbb{R}$, together with some developments on the theory of complete minimal surfaces of finite total curvature in $\mathbb{H}^{2} \times \mathbb{R}$. Most sections finish with a selection of important open problems. See [Mee, DHM] for more open problems in the theory.

A more detailed introduction to the global theory of CMC surfaces in homogeneous 3 -spaces can be found in the Lecture Notes by Daniel, Hauswirth and Mira DHM].

The authors are grateful to H. Rosenberg, B. Daniel and J.A. Gálvez for useful observations about this manuscript.

\section{Homogeneous 3-spaces and Thurston geometries}

Homogeneous spaces are the natural generalization of space forms. By definition, a manifold is said to be homogeneous if the isometry group acts transitively on the manifold. Roughly speaking, the manifold looks the same at all the points, even though, standing at one point, the manifold can look different in different directions. In the simply connected case, the classification of the 3-dimensional homogeneous spaces is well-known. It turns out that any simply connected homogeneous 3-space must have isometry group of dimension 6,4 or 3 . The complete list of these spaces is the following (see subsections below for more details):

- The spaces with 6-dimensional isometry group are the space forms: the Euclidean space $\mathbb{R}^{3}$, the hyperbolic space $\mathbb{H}^{3}(\kappa)$, and the standard sphere $\mathbb{S}^{3}(\kappa)$. For simplicity we will assume that $\kappa= \pm 1$ and write $\mathbb{H}^{3}=\mathbb{H}^{3}(-1)$ and $\mathbb{S}^{3}=\mathbb{S}^{3}(1)$.

- The spaces with 4-dimensional isometry group are fibrations over the 2-dimensional space forms. They are the product spaces $\mathbb{H}^{2} \times \mathbb{R}$ and $\mathbb{S}^{2} \times \mathbb{R}$, the Berger spheres, the Heisenberg space $\mathrm{Nil}_{3}$ and the universal covering of the Lie group $\operatorname{PSL}(2, \mathbb{R})$.

- The spaces with 3-dimensional isometry group are a certain class of Lie groups; among them we specially quote the space $\mathrm{Sol}_{3}$.

These spaces are closely related with Thurston's Geometrization Conjecture. This recently proved conjecture states that any compact orientable 3-manifold can be cut by disjoint embedded 2-spheres or tori into pieces, each one of them, after gluing 2-balls or solid tori along its boundary components, admits a geometric structure. A 3-manifold without boundary is said to admit a geometric structure if it can be endowed with a complete locally homogeneous metric. In this case, by considering its universal covering we obtain a complete simply-connected locally homogeneous space and hence, by a result of Singer, homogeneous. Thus, a 3-manifold admitting a geometric structure can be realized as the quotient of a homogeneous simply connected 3-space under the action of a subgroup of a Lie group acting transitively by isometries. The list of the maximal geometric structures that give compact quotients consists of eight of the previously described spaces: the three space forms, the two product spaces, $\mathrm{Nil}_{3}$, the universal covering of $\operatorname{PSL}(2, \mathbb{R})$ and $\mathrm{Sol}_{3}$ (Berger spheres must be excluded from this list because they are 
not maximal, their isometry group are contained in the one of the standard sphere $\mathbb{S}^{3}$ ). We refer to $\mathrm{Sco}, \mathrm{Bon}$ for more details.

\subsection{Homogeneous spaces with 4-dimensional isometry group.} Denote by $\mathcal{M}^{2}(\kappa)$ the 2-dimensional space form of constant curvature $\kappa$ (for example, $\mathcal{M}^{2}(\kappa)=\mathbb{R}^{2}, \mathbb{H}^{2}, \mathbb{S}^{2}$ for $\kappa=0,-1,1$ respectively). As commented above, any simply connected homogeneous 3-space with 4-dimensional isometry group admits a fibration over $\mathcal{M}^{2}(\kappa)$, for some $\kappa \in \mathbb{R}$. Moreover, these spaces can be parameterized in terms of the base curvature $\kappa$ and the bundle curvature $\tau$, that satisfy $\kappa-4 \tau^{2} \neq 0$. We will use the notation $\mathbb{E}^{3}(\kappa, \tau)$ for these homogeneous spaces.

1. When $\tau=0$, we have the product spaces $\mathcal{M}^{2}(\kappa) \times \mathbb{R}$, i.e. up to scaling, the spaces $\mathbb{S}^{2} \times \mathbb{R}$ when $\kappa>0$, and $\mathbb{H}^{2} \times \mathbb{R}$ when $\kappa<0$.

2. When $\tau \neq 0$ and $\kappa>0$, the corresponding spaces are the Berger spheres, a family of 2-parameter (1-parameter after a homothetical change of coordinates) metrics on the sphere, obtained by deforming the standard metric in such a way that the Hopf fibration is still a Riemannian fibration. They can also be seen as the Lie group $\mathrm{SU}(2)$ endowed with a 1-parameter family of left-invariant metrics.

3. When $\tau \neq 0$ and $\kappa=0, \mathbb{E}^{3}(\kappa, \tau)$ is the Heisenberg group $\mathrm{Nil}_{3}$, the nilpotent Lie group

$$
\left\{\left(\begin{array}{ccc}
1 & a & b \\
0 & 1 & c \\
0 & 0 & 1
\end{array}\right) ; a, b, c \in \mathbb{R}\right\}
$$

endowed with a 1-parameter family of left-invariant metrics, all of them isometrically equivalent after a homothetical change of coordinates.

4. When $\tau \neq 0$ and $\kappa<0$, we obtain the universal covering of the Lie group $\operatorname{PSL}(2, \mathbb{R})$, endowed with a 2-parameter (again 1-parameter after homotheties) family of leftinvariant metrics.

There exists a common setting for all these spaces. Indeed, label $\mathbb{D}(\rho)=\left\{\left(x_{1}, x_{2}\right) \in\right.$ $\left.\mathbb{R}^{2} ; x_{1}^{2}+x_{2}^{2}<\rho^{2}\right\}$. Then, if $\kappa=0$ (resp. $\left.\kappa<0\right)$, the space $\mathbb{E}^{3}(\kappa, \tau)$ can be viewed as $\mathbb{R}^{3}$ (resp. $\mathbb{D}(2 / \sqrt{-\kappa}) \times \mathbb{R})$ endowed with the metric

$$
d s^{2}=\lambda^{2}\left(d x_{1}^{2}+d x_{2}^{2}\right)+\left(\tau \lambda\left(x_{2} d x_{1}-x_{1} d x_{2}\right)+d x_{3}\right)^{2}, \quad \lambda=\frac{1}{1+\frac{\kappa}{4}\left(x_{1}^{2}+x_{2}^{2}\right)} .
$$

Also, for $\kappa>0,\left(\mathbb{R}^{3}, d s^{2}\right)$ corresponds to the universal cover of $\mathbb{E}^{3}(\kappa, \tau)$ minus one fiber. In all cases, up to a homothetical change of coordinates we can suppose without loss of generality that $\kappa-4 \tau^{2}= \pm 1$.

The corresponding Riemannian fibration $\pi: \mathbb{E}^{3}(\kappa, \tau) \rightarrow \mathcal{M}^{2}(\kappa)$ is given here by the projection on the first two coordinates. The unitary vector field

$$
\xi=\frac{\partial}{\partial x_{3}}
$$

is a Killing field tangent to the fibers of $\pi$, and will be referred to as the vertical field of the space $\mathbb{E}^{3}(\kappa, \tau)$. It satisfies the equation

$$
\widehat{\nabla}_{X} \xi=\tau X \times \xi
$$

for all vector fields $X$ in $\mathbb{E}^{3}(\kappa, \tau)$. Here $\widehat{\nabla}$ is the Levi-Civita connection, $\times$ the cross product and $\tau$ the bundle curvature (this is basically the definition of $\tau$ ). 
A remarkable difference between the spaces $\mathbb{E}^{3}(\kappa, \tau)$ is that their isometry group has four connected components in the case $\tau=0$, and only two when $\tau \neq 0$. This follows from the fact that any isometry in the product spaces can either preserve or reverse the orientation of the base and the fibers independently, while in the case $\tau \neq 0$ it can only either preserve or reverse both orientations. In particular, reflections only exist in product spaces.

Also, when $\tau \neq 0$ the spaces $\mathbb{E}^{3}(\kappa, \tau)$ are Lie groups, and if we set $\sigma:=\frac{\kappa}{2 \tau}$, an orthonormal frame of left-invariant vector fields (called the canonical frame) is given by

$$
\begin{aligned}
& E_{1}=\lambda^{-1}\left(\cos \left(\sigma x_{3}\right) \frac{\partial}{\partial x_{1}}+\sin \left(\sigma x_{3}\right) \frac{\partial}{\partial x_{2}}\right)+\tau\left(x_{1} \sin \left(\sigma x_{3}\right)-x_{2} \cos \left(\sigma x_{3}\right)\right) \frac{\partial}{\partial x_{3}} \\
& E_{2}=\lambda^{-1}\left(-\sin \left(\sigma x_{3}\right) \frac{\partial}{\partial x_{1}}+\cos \left(\sigma x_{3}\right) \frac{\partial}{\partial x_{2}}\right)+\tau\left(x_{1} \cos \left(\sigma x_{3}\right)+x_{2} \sin \left(\sigma x_{3}\right)\right) \frac{\partial}{\partial x_{3}} \\
& E_{3}=\xi=\frac{\partial}{\partial x_{3}}
\end{aligned}
$$

2.2. Homogeneous spaces with 3-dimensional isometry group. Of all homogeneous spaces with 3 -dimensional isometry group, $\mathrm{Sol}_{3}$ is specially important, since it is the only Thurston geometry among them. We will now describe some aspects of this space.

A useful representation of $\mathrm{Sol}_{3}$ is the space $\mathbb{R}^{3}$ with the metric

$$
d s^{2}=e^{2 x_{3}} d x_{1}^{2}+e^{-2 x_{3}} d x_{2}^{2}+d x_{3}^{2},
$$

that is left-invariant for the structure of Lie group given by

$$
\left(x_{1}, x_{2}, x_{3}\right) \cdot\left(y_{1}, y_{2}, y_{3}\right)=\left(x_{1}+e^{-x_{3}} y_{1}, x_{2}+e^{x_{3}} y_{2}, x_{3}+y_{3}\right) .
$$

The following vector fields form an orthonormal left-invariant frame

$$
E_{1}=e^{-x_{3}} \frac{\partial}{\partial x_{1}}, \quad E_{2}=e^{x_{3}} \frac{\partial}{\partial x_{2}}, \quad E_{3}=\frac{\partial}{\partial x_{3}} .
$$

The isometries in $\mathrm{Sol}_{3}$ are generated by the three 1-parameter groups of translations

$$
\begin{aligned}
&\left(x_{1}, x_{2}, x_{3}\right) \mapsto\left(x_{1}+c, x_{2}, x_{3}\right), \quad\left(x_{1}, x_{2}, x_{3}\right) \mapsto\left(x_{1}, x_{2}+c, x_{3}\right), \\
&\left(x_{1}, x_{2}, x_{3}\right) \mapsto\left(e^{-c} x_{1}, e^{c} x_{2}, x_{3}+c\right),
\end{aligned}
$$

and by the orientation reversing isometries fixing the origin

$$
\left(x_{1}, x_{2}, x_{3}\right) \mapsto\left(-x_{1}, x_{2}, x_{3}\right), \quad\left(x_{1}, x_{2}, x_{3}\right) \mapsto\left(x_{2},-x_{1},-x_{3}\right)
$$

A remarkable fact is the existence of two canonical foliations, namely

$$
\mathcal{F}_{1}=\left\{x_{1}=\text { constant }\right\}, \quad \mathcal{F}_{2}=\left\{x_{2}=\text { constant }\right\}
$$

whose leaves are totally geodesic surfaces isometric to the hyperbolic plane $\mathbb{H}^{2}$. Reflections across any of these leaves are orientation reversing isometries of $\mathrm{Sol}_{3}$. 


\section{CMC surfaces: basic equations}

In this section we present three important tools for our study. One is the set of integrability equations of CMC surfaces in $\mathbb{E}^{3}(\kappa, \tau)$ by Daniel Dan1. Another one the Abresch-Rosenberg differential, a holomorphic quadratic differential geometrically defined on any CMC surface in $\mathbb{E}^{3}(\kappa, \tau)$. The third one is a local isometric correspondence for CMC surfaces in $\mathbb{E}^{3}(\kappa, \tau)$ via which one can pass from one homogeneous space into another when studying CMC surfaces Dan1]. Some notions about the stability operator of CMC surfaces are also given.

3.1. Integrability equations in $\mathbb{E}^{3}(\kappa, \tau)$. It is well known that the GaussCodazzi equations are the integrability conditions of surface theory in $\mathbb{R}^{3}, \mathbb{S}^{3}$ and $\mathbb{H}^{3}$. In other homogeneous spaces, the situation is more complicated.

Let $\psi: \Sigma \rightarrow \mathbb{E}^{3}(\kappa, \tau)$ be an isometric immersion with unit normal map $\eta$, and consider on $\Sigma$ the conformal structure given by its induced metric via $\psi$. Associated to a conformal parameter $z=s+i t$ on $\Sigma$, we will consider the usual operators $\partial_{z}=\left(\partial_{s}-i \partial_{t}\right) / 2$ and $\partial_{\bar{z}}=\left(\partial_{s}+i \partial_{t}\right) / 2$. Also denote by $\xi$ the vertical Killing field of $\mathbb{E}^{3}(\kappa, \tau)$.

Definition 3.1. We call the fundamental data of $\psi$ the 5-tuple $\left(\lambda|d z|^{2}, u, H, p d z^{2}, A d z\right)$ where $H$ is the mean curvature and

$$
\lambda=2\left\langle\psi_{z}, \psi_{\bar{z}}\right\rangle, \quad u=\langle N, \xi\rangle, \quad p=-\left\langle\psi_{z}, N_{z}\right\rangle, \quad A=\left\langle\xi, \psi_{z}\right\rangle .
$$

The function $u$ is commonly called the angle function of the surface.

Once here, a set of necessary and sufficient conditions for the integrability of CMC surfaces in $\mathbb{E}^{3}(\kappa, \tau)$ can be written in terms of these fundamental data. This is a result by B. Daniel [Dan1, although the formulation that we expose here (i.e. in terms of a conformal parameter on the surface) comes from FeMi2.

Theorem 3.2 (Dan1, FeMi2 $)$. The fundamental data of an immersed surface $\psi: \Sigma \rightarrow$ $\mathbb{E}^{3}(\kappa, \tau)$ satisfy the following integrability conditions:

$$
\left\{\begin{array}{cccc}
(\mathbf{C . 1}) & p_{\bar{z}} & = & \frac{\lambda}{2}\left(H_{z}+u A\left(\kappa-4 \tau^{2}\right)\right) . \\
(\mathbf{C . 2}) & A_{\bar{z}} & = & \frac{u \lambda}{2}(H+i \tau) . \\
(\mathbf{C . 3}) & u_{z} & = & -(H-i \tau) A-\frac{2 p}{\lambda} \bar{A} . \\
(\mathbf{C . 4}) & \frac{4|A|^{2}}{\lambda} & & 1-u^{2} .
\end{array}\right.
$$

Conversely, if $\Sigma$ is simply connected, these equations are also sufficient for the existence of a surface $\psi: \Sigma \rightarrow \mathbb{E}^{3}(\kappa, \tau)$ with fundamental data $\left(\lambda|d z|^{2}, u, H, p d z^{2}, A d z\right)$. This surface is unique up to ambient isometries preserving the orientations of base and fiber of $\mathbb{E}^{3}(\kappa, \tau)$.

We see then that, in the spaces $\mathbb{E}^{3}(\kappa, \tau)$, more equations apart from the GaussCodazzi ones are needed, due to the loss of symmetries. As a matter of fact, (C.1) is the Codazzi equation, while the Gauss equation does not appear (it is deduced from the rest). These new equations evidence the special character of the vertical direction in the $\mathbb{E}^{3}(\kappa, \tau)$ spaces. 
Definition 3.3. The Abresch-Rosenberg differential of the immersion is defined as the quadratic differential on $\Sigma$ given by

$$
Q d z^{2}=\left(2(H+i \tau) p-\left(\kappa-4 \tau^{2}\right) A^{2}\right) d z^{2} .
$$

It is then easy to see by means of (C.2) that the Codazzi equation (C.1) can be rephrased in terms of $Q$ as

$$
Q_{\bar{z}}=\lambda H_{z}+\left(\kappa-4 \tau^{2}\right) \frac{H_{\bar{z}} A^{2}}{(H+i \tau)^{2}} .
$$

Consequently, one has the following theorem, which generalized the classical fact that the Hopf differential is holomorphic for CMC surfaces in $\mathbb{R}^{3}, \mathbb{S}^{3}$ and $\mathbb{H}^{3}$.

Theorem 3.4 (AbRo1, AbRo2]). Qdz $z^{2}$ is a holomorphic quadratic differential on any $C M C$ surface in $\mathbb{E}^{3}(\kappa, \tau)$.

This is a crucial result of the theory, since it allows the use of holomorphic functions in the geometric classification of CMC surfaces in $\mathbb{E}^{3}(\kappa, \tau)$ (see Section 4 and Section 6 , for instance).

An important tool in the description of CMC surfaces in $\mathbb{R}^{3}, \mathbb{S}^{3}$ and $\mathbb{H}^{3}$ is the classical Lawson correspondence. It establishes an isometric one-to-one local correspondence between CMC surfaces in different space forms that allows to pass, for instance, from minimal surfaces in $\mathbb{R}^{3}$ to $H=1$ surfaces in $\mathbb{H}^{3}$.

The Lawson correspondence was generalized by B. Daniel to the context of homogeneous spaces. Indeed, Daniel discovered in Dan1 an isometric local correspondence for CMC surfaces in all the homogeneous spaces $\mathbb{E}^{3}(\kappa, \tau)$, which can be described as follows in terms of the fundamental data defined above.

Theorem 3.5 (Sister correspondence, [Dan1]). Let $\left(\lambda|d z|^{2}, u, H_{1}, p_{1} d z^{2}, A_{1} d z\right)$ be the fundamental data of a simply connected $H_{1}-C M C$ surface in $\mathbb{E}\left(\kappa_{1}, \tau_{1}\right)$, and consider $\kappa_{2}, \tau_{2}, H_{2} \in \mathbb{R}$ so that

$$
\kappa_{2}-4 \tau_{2}^{2}=\kappa_{1}-4 \tau_{1}^{2}, \quad H_{2}^{2}+\tau_{2}^{2}=H_{1}^{2}+\tau_{1}^{2}
$$

Then if we set $\theta \in \mathbb{R}$ given by $H_{2}-i \tau_{2}=e^{i \theta}\left(H_{1}-i \tau_{1}\right)$, the fundamental data given by

$$
\left(\lambda|d z|^{2}, u, H_{2}, p_{2} d z^{2}=e^{-i \theta} p_{1} d z^{2}, A_{2} d z=e^{-i \theta} A_{1} d z\right)
$$

give rise to a (simply connected) $\mathrm{H}_{2}-C M C$ surface in $\mathbb{E}^{3}\left(\kappa_{2}, \tau_{2}\right)$, which is locally isometric to the original one.

Two surfaces related by the above correspondence are called sister surfaces with phase $\theta$. In particular, the corresponding Abresch-Rosenberg differentials of sister surfaces are related by $Q_{2}=e^{-2 i \theta} Q_{1}$. As special cases of this correspondence we obtain the associate family of minimal surfaces in $\mathcal{M}^{2}(\kappa) \times \mathbb{R}$, and a correspondence between minimal surfaces in $\mathrm{Nil}_{3}$ and CMC $\frac{1}{2}$ surfaces in $\mathbb{H}^{2} \times \mathbb{R}$. Generically, and up to ambient isometries and dilations, the family of sister surfaces for a given choice of $(H, \kappa, \tau)$ is a continuous 1parameter family.

There is a natural notion of graph in these spaces. Since $\mathbb{E}^{3}(\kappa, \tau)$ has a canonical fibration over $\mathcal{M}^{2}(\kappa)$ (see Section 2), we will say that an immersed surface $\Sigma$ in $\mathbb{E}^{3}(\kappa, \tau)$ 
is a (local) graph if the projection to the base is a (local) diffeomorphism. The CMCequation for the graph of a function $u=u(x, y)$ is the PDE (see [Lee] )

$$
\frac{2 H}{\delta^{2}}=\frac{\partial}{\partial x}\left(\frac{\alpha}{\omega}\right)+\frac{\partial}{\partial y}\left(\frac{\beta}{\omega}\right),
$$

where

$$
\begin{gathered}
\delta=1+\frac{\kappa}{4}\left(x^{2}+y^{2}\right), \quad \omega=\sqrt{1+\delta^{2}\left(x^{2}+y^{2}\right)}, \\
\alpha=u_{x}+\tau \frac{y}{\delta}, \quad \beta=u_{y}-\tau \frac{x}{\delta} .
\end{gathered}
$$

For instance, a graph $u=u(x, y)$ in $\mathrm{Nil}_{3} \equiv \mathbb{E}^{3}(0, \tau)$ is minimal if and only if it satisfies the elliptic PDE

$$
\left(1+\beta^{2}\right) u_{x x}-2 \alpha \beta u_{x y}+\left(1+\alpha^{2}\right) u_{y y}=0,
$$

where $\alpha:=u_{x}+y / 2$ and $\beta:=u_{y}-x / 2$.

3.2. Stability and index of CMC surfaces. As it is well known, CMC surfaces in Riemannian 3-manifolds appear as the critical points for the area functional associated to variations of the surface with compact support and constant enclosed volume. Equivalently, an immersed surface $S$ has constant mean curvature $H$ if and only if it is a critical point for the functional Area $-2 H$ Vol. The second variation formula for this functional is given by

$$
Q(f, f)=-\int_{S} f \mathcal{L}(f),
$$

where $\mathcal{L}$ is the Jacobi operator (or stability operator) of the surface:

$$
\mathcal{L}=\Delta+\|\mathcal{B}\|^{2}+\operatorname{Ric}(\eta) .
$$

Here $\Delta$ is the Laplacian for the induced metric on the surface, $\mathcal{B}$ is the second fundamental form, $\eta$ is the unit normal vector field, and Ric is the Ricci curvature in the ambient manifold. As a particular case, the Jacobi operator for CMC surfaces in the spaces $\mathbb{E}^{3}(\kappa, \tau)$ can be rewritten (see [Dan1]) as

$$
\mathcal{L}=\Delta-2 K+4 H^{2}+4 \tau^{2}+\left(\kappa-4 \tau^{2}\right)\left(1+u^{2}\right),
$$

being $K$ the Gaussian curvature of the surface and $u$ the angle function (see Definition 3.1). A Jacobi function is a function $f$ for which $\mathcal{L}(f)=0$.

A CMC surface $S$ is said to be stable (resp. weakly stable) if

$$
Q(f, f)=-\int_{S} f \mathcal{L}(f) \geq 0
$$

holds for any smooth function $f$ on $S$ with compact support (resp. with compact support and $\left.\int_{S} f=0\right)$. For instance, CMC graphs in $\mathbb{E}^{3}(\kappa, \tau)$ are stable, and compact CMC surfaces bounding isoperimetric regions are weakly stable (but not necessarily stable, as round spheres in $\mathbb{R}^{3}$ show).

An important concept related to stability is the index of a CMC surface. The index of a compact CMC surface is defined as the number of negative eigenvalues of its Jacobi operator. Thus, stable CMC surfaces (in particular CMC graphs) have index zero. Round spheres in $\mathbb{R}^{3}$ have index one.

We refer to MPR for more details about stability of CMC surfaces. 


\section{Compact CMC surfaces in $\mathbb{E}^{3}(\kappa, \tau)$}

In this section we explain the most important results that are known regarding the existence and uniqueness of compact CMC surfaces in the homogeneous 3 -spaces $\mathbb{E}^{3}(\kappa, \tau)$. The fundamental examples are the rotational CMC spheres, and we shall be interested in their uniqueness among compact embedded CMC surfaces, and among immersed CMC surfaces. These problems are called, respectively, the Alexandrov and Hopf problems.

4.1. Rotational compact CMC surfaces. Although round spheres in the model spaces $\mathbb{R}^{3}, \mathbb{S}^{3}, \mathbb{H}^{3}$ are CMC spheres, this does not hold for the rest of homogeneous spaces. However, in all the spaces $\mathbb{E}^{3}(\kappa, \tau)$ there exist rotations with respect to the vertical axis, and so there is a natural notion of rotational surface. It is hence natural to seek CMC spheres (and CMC tori) in $\mathbb{E}^{3}(\kappa, \tau)$ among the class of rotational surfaces. This can be done by ODE analysis, and the result of this can be summarized as follows:

Theorem 4.1. (Structure of rotational CMC spheres in $\mathbb{E}^{3}(\kappa, \tau)$ ).

1. If $\kappa-4 \tau^{2}>0$, then for every $H \in \mathbb{R}$ there exists a unique rotational CMC $H$ sphere (up to isometries) in $\mathbb{E}^{3}(\kappa, \tau)$. These spheres are embedded if $\tau=0$, i.e. in $\mathbb{S}^{2} \times \mathbb{R}$, and also for most Berger spheres. However, for some Berger spheres with small bundle curvature $\tau$ (with respect to a fixed $\kappa$ ) there is a certain region of variation of the parameters $(H, \tau)$ where the spheres are non-embedded. This region can be explicitly described, see [Tor].

2. If $\kappa-4 \tau^{2}<0$, then

- if $H^{2} \leqslant-\frac{\kappa}{4}$, then there exists no rotational $C M C H$ sphere in $\mathbb{E}^{3}(\kappa, \tau)$,

- if $H^{2}>-\frac{\kappa}{4}$, then there exists a unique rotational CMC $H$ sphere (up to isometries) in $\mathbb{E}^{3}(\kappa, \tau)$. All these spheres are embedded.

Let us remark that all these CMC spheres can be constructed explicitly. We shall call them canonical rotational CMC spheres. For example, the rotational CMC $H$ spheres in $\mathbb{S}^{2} \times \mathbb{R} \subset \mathbb{R}^{4}$ are given by the formula

$$
\psi(u, v)=(-\cos k(u), \sin k(u) \cos v, \sin k(u) \sin v, h(u)),
$$

where $-1 \leq u \leq 1, H \in \mathbb{R}$ and

$$
k(u):=2 \arctan \left(\frac{2 H}{\sqrt{1-u^{2}}}\right), \quad h(u):=\frac{4 H}{\sqrt{4 H^{2}+1}} \operatorname{arcsinh}\left(\frac{u}{\sqrt{1-u^{2}+4 H^{2}}}\right) .
$$

Besides these rotational CMC spheres, there also exist rotational CMC tori in $\mathbb{E}^{3}(\kappa, \tau)$ when (and only when) $\kappa-4 \tau^{2}>0$ (excluding minimal surfaces in $\mathbb{S}^{2} \times \mathbb{R}$ ). For $\mathbb{S}^{2} \times \mathbb{R}$, they are all embedded (see Pedrosa [Ped]). For Berger spheres the situation is explained by Torralbo and Urbano in Tor, ToUr]; one has for every $H$ rotational embedded CMC tori given by the Hopf lift of a circle in $\mathbb{S}^{2}$, but there also exist some other non-flat rotational CMC tori. The embeddedness problem for such tori is open in general, but for the minimal case there are embedded rotational tori other than Clifford tori. This contrasts with the case of embedded minimal tori in $\mathbb{S}^{3}$.

A general study of CMC surfaces in $\mathbb{H}^{2} \times \mathbb{R}$ and $\mathbb{S}^{2} \times \mathbb{R}$ invariant by a continuous 1-parameter subgroup of ambient isometries can be found in $\mathrm{SaE}, \mathrm{SaT}$ ]. 
4.2. The Alexandrov problem in $\mathbb{E}^{3}(\kappa, \tau)$. One of the fundamental theorems of CMC surface theory is the so-called Alexandrov theorem.

Theorem 4.2 (Alexandrov). Any compact embedded CMC surface in $\mathbb{R}^{3}, \mathbb{H}^{3}$ or a hemisphere of $\mathbb{S}^{3}$ is a round sphere.

Proof. The proof relies on the so-called Alexandrov reflection principle, which we sketch for $\mathbb{R}^{3}$ although it works with great generality. Consider a plane $P$ disjoint from the compact embedded CMC surface $\Sigma$, and start translating it in a parallel way towards $\Sigma$. After it first touches $\Sigma$, we start reflecting the piece of $\Sigma$ that has been left behind across this new translated plane. In this way we will eventually reach a first contact point with the unreflected part of $\Sigma$. By the maximum principle for elliptic PDEs, this means that $\Sigma$ is symmetric with respect to such a plane. As the starting plane was arbitrary, the compact surface must be a round sphere.

It must be emphasized that there exist embedded CMC tori in $\mathbb{S}^{3}$, such as the product tori $\mathbb{S}^{1}(r) \times \mathbb{S}^{1}\left(\sqrt{1-r^{2}}\right) \subset \mathbb{S}^{3}$. Thus, the hemisphere hypothesis is necessary in the case of $\mathbb{S}^{3}$.

Motivated by this result, the problem of classifying all compact embedded CMC surfaces in a Riemannian 3-manifold $\bar{M}^{3}$ will be called the Alexandrov problem in $\bar{M}^{3}$.

In the case of CMC surfaces in the product spaces $\mathbb{H}^{2} \times \mathbb{R}$ and $\mathbb{S}^{2} \times \mathbb{R}$, the Alexandrov technique can be applied for horizontal directions, and so the following result holds.

Theorem 4.3 (Hsiang-Hsiang). Any compact embedded CMC surface in $\mathbb{H}^{2} \times \mathbb{R}$ or $\mathbb{S}_{+}^{2} \times \mathbb{R}$ is a standard rotational CMC sphere.

Again, the hemisphere hypothesis is necessary, since we know that there are embedded CMC tori in $\mathbb{S}^{2} \times \mathbb{R}$.

As regards the homogeneous spaces $\mathbb{E}^{3}(\kappa, \tau)$ with $\tau \neq 0$, i.e. Heisenberg space, Berger spheres and the universal covering of $\mathrm{SL}_{2}(\mathbb{R})$, the Alexandrov problem is open. The main difficulty there is that these spaces do not admit reflections, and hence the reflection principle does not hold.

4.3. The Hopf problem in $\mathbb{E}^{3}(\kappa, \tau)$. Another fundamental result of CMC surface theory is the Hopf theorem:

Theorem 4.4 (Hopf). Any immersed CMC sphere in $\mathbb{R}^{3}, \mathbb{S}^{3}$ or $\mathbb{H}^{3}$ is a round sphere.

Proof. The Hopf differential (see Section 3) of any CMC surface in $\mathbb{R}^{3}, \mathbb{S}^{3}$ or $\mathbb{H}^{3}$ is holomorphic, and vanishes at the umbilical points of the surface. As any holomorphic quadratic differential must vanish on the Riemann sphere, we conclude that immersed CMC spheres are totally umbilical, and hence round spheres.

The Hopf problem in a Riemannian 3-manifold $\bar{M}^{3}$ will refer to the problem of classifying all immersed CMC spheres in $\bar{M}^{3}$.

As was proved in Theorem 3.4. CMC surfaces in the homogeneous spaces $\mathbb{E}^{3}(\kappa, \tau)$ have an associated holomorphic quadratic differential: the Abresch-Rosenberg differential $Q_{A R}$. This allows to solve the Hopf problem in $\mathbb{E}^{3}(\kappa, \tau)$, along the lines suggested by Hopf's classical theorem. We present here an alternative proof to the original one by Abresch and Rosenberg AbRo1, AbRo2, based on Daniel's integrability equations, and on some ideas in [FeMi2, GMM] (see dCF, EsRo, DHM]). 
Theorem 4.5 (Abresch-Rosenberg). Any immersed $C M C$ sphere in $\mathbb{E}^{3}(\kappa, \tau)$ is a standard rotational sphere.

Proof. As the Abresch-Rosenberg $Q_{A R}$ is holomorphic, it must vanish on any immersed CMC sphere. So, we need to prove that spheres with $Q_{A R}=0$ are rotational.

First, one can observe that on any CMC surface in $\mathbb{E}^{3}(\kappa, \tau)$, the equation $Q_{A R}=0$ together with the integrability conditions in Theorem 3.2 imply that the function $w:=$ $\operatorname{arctanh}(u)$ is a harmonic function on the surface (here $u$ is the angle function of the surface). So, once we rule out the case $u=$ const. which does not produce CMC spheres (except for slices in $\mathbb{S}^{2} \times \mathbb{R}$ ), we can define $\zeta$ to be a local conformal parameter on the surface with $\operatorname{Re} \zeta=w$. Again from the integrability equations (C.1) to (C.4) we see that all fundamental data of the surface depend only on $w$ (and not on $\operatorname{Im} \zeta$ ). This implies that the surface is a local piece of some CMC surface invariant by a continuous 1-parameter subgroup of ambient isometries of $\mathbb{E}^{3}(\kappa, \tau)$.

If the surface is compact, this isometry subgroup must be the group of rotations around the vertical axis, with the possible exception of Berger 3-spheres (the only space in which there are non-rotational compact continuous isometry subgroups). However, it is clear that any element of such a non-rotational isometry subgroup has no fixed points. Hence, by the invariance property, there is a globally defined non-zero vector field on the surface (that is tangent to the orbits). But this is impossible on a sphere. Hence, the isometry subgroup is always the group of rotations around the vertical axis, and thus the CMC sphere is rotational, as wished.

4.4. The isoperimetric problem in $\mathbb{E}^{3}(\kappa, \tau)$. The Alexandrov problem is very relevant to the isoperimetric problem in a Riemannian 3-manifold $\bar{M}^{3}$; indeed, any solution to the isoperimetric problem in $\bar{M}^{3}$ is a region bounded by a compact embedded CMC surface. So, for instance, the only candidates to solve the isoperimetric problem for a given volume in $\mathbb{H}^{2} \times \mathbb{R}$ are rotational CMC spheres. Another geometric property satisfied by isoperimetric solutions is that they are weakly stable, see Section 2.

The class of isoperimetric solutions in $\mathbb{R}^{3}, \mathbb{S}^{3}$ and $\mathbb{H}^{3}$ is the class of round spheres. The isoperimetric problem in $\mathbb{S}^{2} \times \mathbb{R}$ and $\mathbb{H}^{2} \times \mathbb{R}$ has been also explicitly solved, as follows:

1. The isoperimetric regions in $\mathbb{H}^{2} \times \mathbb{R}$ are exactly the regions bounded by the canonical rotational CMC spheres. (Hsiang-Hsiang).

2. There is a value $H_{1} \approx 0.33$ such that the isoperimetric regions in $\mathbb{S}^{2} \times \mathbb{R}$ are exactly the regions bounded by the canonical rotational CMC $H$ spheres with $H \geq H_{1}$. (Pedrosa).

So, regading complete simply connected Riemannian 3-manifolds, the isoperimetric problem is fully solved in $\mathbb{R}^{3}, \mathbb{S}^{3}, \mathbb{H}^{3}, \mathbb{S}^{2} \times \mathbb{R}$ and $\mathbb{H}^{2} \times \mathbb{R}$. A remarkable advance in this direction has been obtained very recently by F. Torralbo and F. Urbano ToUr, who have added to this list a certain subfamily of Berger spheres:

Theorem 4.6 (Torralbo-Urbano). The solutions to the isoperimetric problem in the Berger spheres $\mathbb{E}^{3}(\kappa, \tau)$ with $\frac{1}{3} \leq \frac{4 \tau^{2}}{\kappa}<1$ are the canonical rotational CMC spheres.

The proof of this result relies on embedding the Berger spheres $\mathbb{E}^{3}(\kappa, \tau)$ into the 4dimensional complex space $\mathbb{C} P^{2}$, and using a Willmore inequality in this space due to Montiel and Urbano $\mathrm{MoUr}$.

For the rest of the spaces, the isoperimetric problem is open. In any case, the general theory of the isoperimetric problem together with the Abresch-Rosenberg uniqueness 
theorem imply that, for small volumes, the isoperimetric solutions are canonical rotational CMC spheres with large $H$.

4.5. Open problems. One of the major unsolved problems in the theory is the Alexandrov problem when $\tau \neq 0$, i.e. in $\mathrm{Nil}_{3}$, the universal cover of $\operatorname{PSL}(2, \mathbb{R})$ and $\operatorname{Berger}$ hemispheres. It is conjectured that canonical rotational spheres are the only compact embedded CMC surfaces in these spaces. A related open problem is the isoperimetric problem in $\mathrm{Nil}_{3}$, the universal cover of $\operatorname{PSL}(2, \mathbb{R})$ and the Berger spheres not covered by Theorem 4.6. In the first two cases, it is conjectured that the isoperimetric solutions are exactly the canonical rotational spheres.

Besides, it is conjectured by Nelli and Rosenberg [NeRo2] that compact weakly stable CMC surfaces in $\mathbb{H}^{2} \times \mathbb{R}$ are rotational CMC spheres.

Another important problem of the theory is the construction of higher genus compact (immersed) CMC surfaces, (e.g. CMC tori) in $\mathbb{E}^{3}(\kappa, \tau)$ with $\kappa \leq 0$.

\section{CMC spheres in $\mathrm{Sol}_{3}$}

In this section we will expose the recent solution to the Alexandrov problem (i.e. the classification of compact embedded CMC surfaces) and the Hopf problem (i.e. the classification of immersed CMC spheres) in the remaining Thurston 3-geometry: the homogeneous space $\mathrm{Sol}_{3}$.

The first step in this direction is that we can solve the Alexandrov problem from a topological point of view.

Theorem 5.1 (Rosenberg). Any compact embedded CMC surface in $\mathrm{Sol}_{3}$ is, topologically, a sphere.

Proof. By Alexandrov reflection principle using the two canonical foliations of $\mathrm{Sol}_{3}$ (recall that reflections across their leafs are orientation-reversing isometries of $\mathrm{Sol}_{3}$ ), it turns out that any compact embedded CMC surface in $\mathrm{Sol}_{3}$ is a bi-graph with respect to two linearly independent directions in $\mathbb{R}^{3}$. Thus, the surface is, topologically, a sphere.

This result leaves us with the problem of classifying (embedded) CMC spheres. A substantial difficulty for this task is that $\mathrm{Sol}_{3}$ has no rotations. Hence, there are no rotational CMC spheres to use in order to gain insight of the theory, and even the existence of CMC spheres for a given value of $H$ needs to be settled.

The next theorem is the main result of the section, and solves the Hopf and Alexandrov problems in $\mathrm{Sol}_{3}$.

Theorem 5.2 (Daniel-Mira, Meeks). For every $H>0$ there exists an embedded $C M C$ $H$ sphere $S_{H}$ in $\mathrm{Sol}_{3}$. This sphere is unique in the following sense:

1. Hopf uniqueness: every immersed $C M C H$ sphere in $\mathrm{Sol}_{3}$ is a left-translation of $S_{H}$.

2. Alexandrov uniqueness: every compact embedded $C M C H$ surface in $\mathrm{Sol}_{3}$ is a lefttranslation of $S_{H}$.

Moreover, each sphere $S_{H}$ has index one, it inherits all possible symmetries of the ambient space (its group of ambient isometries is the diedral group $D_{4}$ ), its Lie group Gauss map is a diffeomorphism, and the family $\left\{S_{H}: H>0\right\}$ is real analytic (up to left translations). 
Remark 5.3. Theorem[5.2 was obtained by Daniel and Mira [DaMi] for $H>1 / \sqrt{3}$. For the remaining values $H \in(0,1 / \sqrt{3}]$, Daniel and Mira also proved the uniqueness in the Hopf and Alexandrov sense for all values of $H$ for which there exists an index one CMC $H$ sphere. Finally, Meeks [Mee] obtained the existence of index one CMC H spheres for every $H>0$ (and not just for $H>1 / \sqrt{3}$ ). This concluded the proof of Theorem 5.2.

We shall split the sketch of the proof of Theorem 5.2 into two parts.

5.1. Proof of Theorem 5.2: uniqueness. The results of this part are contained in DaMi. The Lie group Gauss map $g: \Sigma \rightarrow \overline{\mathbb{C}}$ of a CMC surface $X: \Sigma \rightarrow$ Sol $_{3}$ satisfies the following elliptic PDE (here $z$ is a conformal parameter on the surface):

$$
g_{z \bar{z}}=A(g) g_{z} g_{\bar{z}}+B(g) g_{z} \bar{g}_{\bar{z}}
$$

where, by definition,

$$
\begin{gathered}
A(q)=\frac{R_{q}}{R}=\frac{2 H\left(1+|q|^{2}\right) \bar{q}+2 q}{R(q)}, \quad B(q)=\frac{R_{\bar{q}}}{R}-\frac{\bar{R}_{\bar{q}}}{\bar{R}}=-\frac{4 H\left(1+|q|^{2}\right)\left(\bar{q}+q^{3}\right)}{|R(q)|^{2}}, \\
R(q)=H\left(1+|q|^{2}\right)^{2}+q^{2}-\bar{q}^{2} .
\end{gathered}
$$

Moreover, the surface $X$ is uniquely determined by the Gauss map $g$, and it can actually be recovered from $g$ by means of an integral representation formula.

Once here, the first idea in order to prove a Hopf-type theorem is to look for a holomorphic quadratic differential for $\mathrm{CMC}$ surfaces in $\mathrm{Sol}_{3}$. However, it seems that such a holomorphic object is not available in the theory; this constitutes another key difference from the theory of CMC surfaces in the other Thurston 3-geometries exposed in the previous section, where the Abresch-Rosenberg (or the Hopf differential) is holomorphic.

Still, it is not strictly necessary to obtain a holomorphic differential in order to prove a Hopf-uniqueness theorem: it suffices to find a geometrically defined quadratic differential with isolated zeros of negative index, so that it vanishes identically on spheres. This is done as follows.

Theorem 5.4 (Daniel-Mira). Let $H>0$, and assume that there exists an index one $C M C H$ sphere $S_{H}$ in $\mathrm{Sol}_{3}$. Then there exists a quadratic differential $Q_{H}$, geometrically defined on any $C M C H$ surface in $\mathrm{Sol}_{3}$, with the following properties:

1. It only has isolated zeros of negative index (thus, it vanishes on spheres).

2. $Q_{H}=0$ holds for a surface $X: \Sigma \rightarrow \mathrm{Sol}_{3}$ if and only if $X$ is a left-translation of some piece of the sphere $S_{H}$.

Moreover, the sphere $S_{H}$ is embedded, and it is therefore unique in $\mathrm{Sol}_{3}$ (up to lefttranslations) in the Hopf sense and in the Alexandrov sense.

The quadratic differential $Q_{H}$ is constructed as follows. Let $G: S_{H} \equiv \overline{\mathbb{C}} \rightarrow \overline{\mathbb{C}}$ denote the Gauss map of $S_{H}$. Then $G$ is a diffeomorphism (otherwise one can construct a Jacobi function $u$ on $S_{H}$ with $u(p)=\nabla u(p)=0$ at some $p \in S_{H}$, which contradicts the index one condition by Courant's nodal domain theorem).

Once here, the differential $Q_{H}$ is defined for any CMC $H$ surface $X: \Sigma \rightarrow \mathrm{Sol}_{3}$ with Gauss map $g: \Sigma \rightarrow \overline{\mathbb{C}}$ by

$$
Q_{H}=\left(L(g) g_{z}^{2}+M(g) g_{z} \bar{g}_{z}\right) d z^{2},
$$


where by definition

$$
M(q)=\frac{1}{R(q)}=\frac{1}{H\left(1+|q|^{2}\right)^{2}+q^{2}-\bar{q}^{2}}
$$

and $L: \overline{\mathbb{C}} \rightarrow \mathbb{C}$ is implicitly given in terms of the Gauss map $G$ of $S_{H}$ by

$$
L(G(z))=-\frac{M(G(z)) \bar{G}_{z}(z)}{G_{z}(z)} .
$$

It must also be emphasized that, by this uniqueness theorem, any index one CMC sphere $S_{H}$ in $\mathrm{Sol}_{3}$ is as symmetric as the ambient space allows: there is a point $p \in \mathrm{Sol}_{3}$ such that $S_{H}$ is invariant with respect to all the isometries of $\mathrm{Sol}_{3}$ that leave $p$ fixed.

\subsection{Proof of Theorem 5.2; existence. Let us define}

$$
\mathcal{I}:=\left\{H>0 \text { : exists an index one CMC } H \text { sphere } S_{H} \text { in } \operatorname{Sol}_{3}\right\}
$$

We prove next the theorem by Meeks Mee that $\mathcal{I}=(0, \infty)$. (The fact that $(1 / \sqrt{3}, \infty) \subset$ $\mathcal{I}$ had been previously obtained in DaMi]).

That $\mathcal{I} \neq \emptyset$ follows from the existence of isoperimetric spheres, which in $\mathrm{Sol}_{3}$ must have index one. That $\mathcal{I}$ is open was proved in DaMi, and follows from the implicit function theorem and from the continuity of the eigenvalues and eigenspaces in the deformation.

The proof that $\mathcal{I}$ is closed is the critical step. The key point is to prevent that the diameters of a sequence of CMC $H_{n}$ spheres $\left(S_{H_{n}}\right)$ with $H_{n} \rightarrow H_{0}>0$ tend to $\infty$. This was proved first by Daniel-Mira, but only for $H_{0}>1 / \sqrt{3}$. The final proof for every $H_{0}>0$ was recently given by Meeks Mee, using the following height estimate: there exists a constant $K\left(H_{0}\right)$ such that for any $C M C H_{0}$ graph (possibly non-compact) with respect to one of the two canonical foliations of $\mathrm{Sol}_{3}$, and with boundary on a leaf, the maximum height attained by the graph with respect to this leaf is $\leq K\left(H_{0}\right)$.

Once this height estimate is ensured, Meeks concludes the proof by some elliptic theory and stability arguments.

5.3. Open problems. Are $\mathrm{CMC}$ spheres in $\mathrm{Sol}_{3}$ weakly stable? Do they all bound isoperimetric regions in $\mathrm{Sol}_{3}$ ? A positive answer is conjectured in DaMi]. What happens in other homogeneous 3-spaces with 3-dimensional isometry group?

It seems very interesting to develop a global theory of minimal surfaces in $\mathrm{Sol}_{3}$. Some natural problems would be proving half-space theorems, classifying entire minimal graphs, or finding properly embedded minimal surfaces of non-trivial topology.

\section{Surfaces of critical CMC}

As we saw in Section $3, \mathrm{CMC} H$ spheres in the homogeneous space $\mathbb{E}^{3}(\kappa, \tau)$ exist exactly for the values $H^{2}>-\kappa / 4$. Besides, one can easily see that there exist entire

rotational CMC $H$ graphs in $\mathbb{E}^{3}(\kappa, \tau), \kappa \leq 0$, whenever $H^{2} \leq-\kappa / 4$. From these results and the maximum principle, we obtain

Theorem 6.1. Any compact CMC H surface in $\mathbb{E}^{3}(\kappa, \tau)$ satisfies $H^{2}>-\kappa / 4$. Also, any entire $C M C$ graph in $\mathbb{E}^{3}(\kappa, \tau), \kappa \leq 0$, satisfies $H^{2} \leq-\kappa / 4$. 
There are several other properties that make the theory of CMC surfaces with $H^{2}>$ $-\kappa / 4$ quite different from the theory of CMC surfaces with $H^{2} \leq-\kappa / 4$. For instance:

1. A properly embedded CMC surface in $\mathbb{H}^{2} \times \mathbb{R}$ with $H>1 / 2$ and finite topology cannot have exactly one end (Espinar, Gálvez, Rosenberg, [EGR]).

2. There exist horizontal and vertical height estimates for CMC surfaces with $H>1 / 2$ in $\mathbb{H}^{2} \times \mathbb{R}$ NeRo2, AEG1, EGR].

3. There are no complete stable CMC surfaces in $\mathbb{H}^{2} \times \mathbb{R}$ with $H>1 / \sqrt{3}$, and the result is expected for $H>1 / 2$. (Nelli-Rosenberg, [NeRo2]). Besides, there are no complete stable CMC surfaces with $H>1 / 2$ in $\mathbb{H}^{2} \times \mathbb{R}$ of parabolic conformal structure (Manzano-Pérez-Rodríguez, MaPR]).

It is hence natural to introduce the following definition.

Definition 6.2. We say that a CMC surface in $\mathbb{E}^{3}(\kappa, \tau)$ with $\kappa \leq 0$ has critical $C M C$ if its mean curvature $H$ satisfies $H^{2}=-\kappa / 4$.

The critical mean curvature is the largest value of $|H|$ for which compact CMC surfaces do not exist. Therefore we have $H=1 / 2$ surfaces in $\mathbb{H}^{2} \times \mathbb{R}$, minimal surfaces in $\mathrm{Nil}_{3}$, and $H=\sqrt{-\kappa} / 2$ surfaces in the universal covering of $\operatorname{PSL}(2, \mathbb{R})$. A remarkable property is that the sister correspondence preserves the property of having critical $\mathrm{CMC}$, and that every simply connected surface of critical CMC is the sister surface of some minimal surface in $\mathrm{Nil}_{3}$.

In this section we will study the global geometry of surfaces with critical CMC, focusing on the existence of harmonic Gauss maps and the classification of entire graphs.

6.1. Harmonic Gauss maps. A smooth map $G: M \rightarrow N$ between Riemannian manifolds is harmonic if it is a critical point for the total energy functional. When $M$ is a surface, harmonicity is a conformal invariant, and it implies that the quadratic differential

$$
Q_{0} d z^{2}=\left\langle G_{z}, G_{z}\right\rangle d z^{2}
$$

is holomorphic, where $z$ is a conformal parameter on $\Sigma$, and $\langle$,$\rangle denotes the metric in N$ (see [FoWo]). We call $Q_{0} d z^{2}$ the Hopf differential associated to $G$.

The Gauss map of CMC surfaces in $\mathbb{R}^{3}$ is harmonic into $\mathbb{S}^{2}$, and its Hopf differential agrees (up to a constant) with the Hopf differential of the surface. Moreover, the CMC surface can be recovered from the Gauss map by a representation formula. This Gauss map opens the door to the use of strong techniques from harmonic maps in the description of CMC surfaces.

The same holds for spacelike CMC surfaces in Minkowski 3-space $\mathrm{E}^{3}$, but this time the harmonic Gauss map takes values into $\mathbb{H}^{2}$. Let us briefly comment this case, since it will play an important role in the development of the section. Let $f: \Sigma \rightarrow \mathrm{E}^{3}$ be a connected spacelike CMC surface, oriented so that its Gauss map $G$ takes values in $\mathbb{H}^{2}$. Here $\mathrm{E}^{3}$ is $\mathbb{R}^{3}$ with the metric $d x^{2}+d y^{2}-d z^{2}$ and $\mathbb{H}^{2}$ is realized in $\mathrm{E}^{3}$ in the usual way. It turns out that $G$ is harmonic into $\mathbb{H}^{2}$ and its associated Hopf differential agrees (up to a multiplicative constant) with the Hopf differential of the immersion $f$. Moreover, the metric of the CMC surface, $\langle d f, d f\rangle=\tau_{0}|d z|^{2}$, is related with $G$ by

$$
2\left\langle G_{z}, G_{\bar{z}}\right\rangle=\frac{\tau_{0}}{4}+\frac{4\left|Q_{0}\right|^{2}}{\tau_{0}} .
$$


Definition 6.3. We will say that a harmonic map $G$ into $\mathbb{H}^{2}$ admits Weierstrass data $\left\{Q_{0}, \tau_{0}\right\}$ if the pullback metric induced by $G$ can be written as

$$
\langle d G, d G\rangle=Q_{0} d z^{2}+\mu|d z|^{2}+\bar{Q}_{0} d \bar{z}^{2}, \quad \mu=\frac{\tau_{0}}{4}+\frac{4\left|Q_{0}\right|^{2}}{\tau_{0}},
$$

$\tau_{0}$ being a positive smooth function.

6.1.1. $\boldsymbol{H}=1 / 2$ surfaces in $\mathbb{H}^{2} \times \mathbb{R}$. We will regard $\mathbb{H}^{2} \times \mathbb{R}=\mathbb{E}^{3}(-1,0)$ in its Minkowski model, i.e.

$$
\mathbb{H}^{2} \times \mathbb{R}=\left\{\left(x_{0}, x_{1}, x_{2}, x_{3}\right): x_{0}>0,-x_{0}^{2}+x_{1}^{2}+x_{2}^{2}=-1\right\} \subset \mathrm{E}^{3} \times \mathbb{R}=\mathrm{E}^{4} .
$$

Using this model, the unit normal vector $\eta$ of an immersed surface $\psi=(N, h): \Sigma \rightarrow$ $\mathbb{H}^{2} \times \mathbb{R}$ takes values in the de Sitter 3-space, and $\{\eta, N\}$ is an orthonormal frame for the Lorentzian normal bundle of $\psi$ in $\mathrm{E}^{4}$. Moreover, if $u$ is the angle function of the surface (that is, the last coordinate of $\eta$ ) and we assume that $u \neq 0$ (that is, that $\psi$ is nowhere vertical, or equivalently, that it is a multigraph), then we can write

$$
\frac{1}{u}(\eta+N)=(G, 1)
$$

for a certain map $G: \Sigma \rightarrow \mathbb{H}^{2}$.

Definition 6.4 ([FeMi1] ). The map G given by (12) will be called the hyperbolic Gauss map of an immersed (nowhere vertical) surface in $\mathbb{H}^{2} \times \mathbb{R}$.

The main property of the hyperbolic Gauss map is the following [FeMi1]:

Theorem 6.5 (Fernández-Mira). The hyperbolic Gauss map of a CMC surface with $H=1 / 2$ in $\mathbb{H}^{2} \times \mathbb{R}$ is a harmonic map into $\mathbb{H}^{2}$, and admits Weierstrass data $\left\{-Q, \lambda u^{2}\right\}$, where $Q d z^{2}, \lambda|d z|^{2}$ and $u$ are, respectively, the Abresch-Rosenberg differential, the metric, and the angle function of the surface.

Conversely, if $\Sigma$ is simply connected, any harmonic map $G: \Sigma \rightarrow \mathbb{H}^{2}$ admitting Weierstrass data is the hyperbolic Gauss map of some $H=1 / 2$ surface in $\mathbb{H}^{2} \times \mathbb{R}$.

Moreover, the space of $H=1 / 2$ surfaces in $\mathbb{H}^{2} \times \mathbb{R}$ with the same hyperbolic Gauss map $G$ is generically two-dimensional, and it can be recovered from $G$ by a representation formula.

The proof of the direct part of the above result follows from equations (2) and the very definition of $G$. The converse part is an integrability argument. This result is of great importance for the rest of this section, since it allows the use of harmonic maps in the description of surfaces of critical CMC.

6.1.2. Minimal surfaces in $\mathbf{N i l}_{3}$. The existence of this harmonic Gauss map for $H=1 / 2$ surfaces in $\mathbb{H}^{2} \times \mathbb{R}$ was extended by B. Daniel Dan2 to the case of minimal surfaces in $\mathrm{Nil}_{3}=\mathbb{E}^{3}\left(0, \frac{1}{2}\right)$.

This time, the harmonic Gauss map is given by the Lie group Gauss map of the surface. Indeed, if we identify the Lie algebra of $\mathrm{Nil}_{3}$ with the tangent space at a point by left multiplication, we can stereographically project the unit normal vector field to obtain a map taking values in the extended complex plane. More specifically, we will consider the model of $\mathrm{Nil}_{3}$ given in Section 2 and its canonical frame of left-invariant 
fields $\left\{E_{1}, E_{2}, E_{3}\right\}$. If $N=\sum N_{i} E_{i}$ is the unit normal of $X: \Sigma \rightarrow \mathrm{Nil}_{3}$, then the Gauss map of $X$ is given by

$$
g=\frac{N_{1}+i N_{2}}{1+N_{3}}: \Sigma \rightarrow \overline{\mathbb{C}}
$$

Now, if the surface is nowhere vertical we can orient it so that $u=\left\langle N, E_{3}\right\rangle$ is positive, and so $g$ takes values in the unit disc $\mathbb{D}$. By identifying $\mathbb{H}^{2}$ with $\left(\mathbb{D}, d s_{P}^{2}\right)$, where $d s_{P}^{2}$ is the Poincaré metric, Daniel obtained in Dan2]:

Theorem 6.6 (Daniel). The Gauss map of a nowhere vertical minimal surface is harmonic into $\mathbb{H}^{2}$.

Conversely, let $g: \Sigma \rightarrow \mathbb{H}^{2}$ be a harmonic map defined on a simply connected oriented Riemann surface into $\mathbb{H}^{2}$, and assume that $g$ is nowhere antiholomorphic (i.e., $g_{z}$ does not vanish at any point). Take $z_{0} \in \Sigma$ and $X_{0} \in \mathrm{Nil}_{3}$.

Then there exists a unique conformal nowhere vertical minimal immersion $X: \Sigma \rightarrow$ $\mathrm{Nil}_{3}$ with $X\left(z_{0}\right)=X_{0}$ having $g$ as its Gauss map. Moreover, $X$ can be uniquely recovered from $g$ through an adequate representation formula.

Furthermore, it can be checked that the Weierstrass data of $g$ as above are $\left\{-Q, \lambda u^{2}\right\}$, where $Q d z^{2}, \lambda|d z|^{2}$ and $u$ are, respectively, the Abresch-Rosenberg differential, the metric, and the angle function of the surface defined in Section 2.

As we saw in Section 3, minimal surfaces in $\mathrm{Nil}_{3}$ and $H=1 / 2$ surfaces in $\mathbb{H}^{2} \times \mathbb{R}$ are related by the sister correspondence, and sister surfaces have the same metric and angle function (in particular, the condition of being nowhere vertical is preserved). As in this case the sister surfaces have opposite Abresch-Rosenberg differentials, it turns out that their respective harmonic Gauss maps are conjugate to each other.

The relation between minimal surfaces in $\mathrm{Nil}_{3}$ and $H=1 / 2$ surfaces in $\mathbb{H}^{2} \times \mathbb{R}$ can be made more explicit by means of the theory of spacelike CMC surfaces in $\mathrm{E}^{3}$, as follows.

Theorem 6.7 (FeMi3]). Let $X=(F, t): \Sigma \rightarrow \mathrm{Nil}_{3}$ be a simply connected nowhere vertical minimal surface with metric $\lambda|d z|^{2}$ and angle function $u$, and $\psi=(N, h): \Sigma \rightarrow$ $\mathbb{H}^{2} \times \mathbb{R}$ its sister surface.

Then $f:=(F, h): \Sigma \rightarrow E^{3}$ is a spacelike $H=1 / 2$ surface in the Minkowski 3 -space with metric $\lambda u^{2}|d z|^{2}$ and Hopf differential-Qdz $z^{2}$, where $Q d z^{2}$ is the Abresch-Rosenberg differential of $X$.

6.1.3. CMC $\sqrt{-\kappa} / 2$ surfaces in $\widehat{\operatorname{PSL}(2, \mathbb{R})}$. In a forthcoming paper [DFM], the authors and B. Daniel will prove that there exists also a harmonic Gauss map for critical CMC surfaces in the remaining case, i.e. the universal covering of the group $\operatorname{PSL}(2, \mathbb{R})$, and will derive a representation formula for them.

6.2. Half-space theorems. One of the most important results in the global study of minimal surfaces in $\mathbb{R}^{3}$ is the classical half-space theorem by Hoffman and Meeks HoMe. This theorem says that any properly immersed minimal surface in $\mathbb{R}^{3}$ lying in a half-space must be a plane parallel to the one determining the half-space. The main tools used here are the maximum principle and the existence of catenoids, a 1-parameter family of minimal surfaces converging to a doubly-covered punctured plane $P$, and intersecting the planes parallel to $P$ in compact curves.

The analogous version for CMC one half surfaces in $\mathbb{H}^{2} \times \mathbb{R}$ was proved in [HRS]. In this setting, horocylinders play the role of the planes in $\mathbb{R}^{3}$. 
Theorem 6.8 (Hauswirth-Rosenberg-Spruck). The only properly immersed CMC one half surfaces in $\mathbb{H}^{2} \times \mathbb{R}$ that are contained in the mean convex side of a horocylinder $C$ are the horocylinders parallel to $C$.

Also, the only properly embedded $C M C$ one half surfaces in $\mathbb{H}^{2} \times \mathbb{R}$ containing a horocylinder in its mean convex side are the horocylinders.

Proof. The main point here is to construct a family of CMC one half surfaces in $\mathbb{H}^{2} \times \mathbb{R}$ to be used in the same way as catenoids in the proof of the half-space theorem in $\mathbb{R}^{3}$. This is achieved by means of compact annuli with boundaries, contained between two horocylinders.

For the case of $\mathrm{Nil}_{3}$, we must distinguish between horizontal and vertical half-spaces. The equivalent to the half-space theorem for surfaces lying in a horizontal half-space is proved by using the family of rotational annuli AbRo2. The corresponding vertical version has been obtained in $\mathrm{DaHa}$, by constructing first a family of horizontal catenoids, i.e. properly embedded minimal annuli (non-rotational) with a geometric behaviour good enough to apply the Hoffman-Meeks technique.

Theorem 6.9 (Daniel-Hauswirth). The only properly immersed minimal surfaces in $\mathrm{Nil}_{3}$ that are contained in a vertical half space are the vertical planes parallel to the one determining the half-space.

Proof. Using the representation formula for minimal surfaces in $\mathrm{Nil}_{3}$ (see Theorem 6.6), it is possible to construct horizontal catenoids in $\mathrm{Nil}_{3}$. These surfaces are a 1-parameter family of properly embedded minimal annuli, intersecting vertical planes $\left\{x_{2}=c\right\}$ in a non-empty closed convex curve. Moreover, the family converges to a double covering of $\left\{x_{2}=0\right\}$ minus a point. They are obtained by integrating a family of harmonic maps that belong to a more general family used in the construction of Riemann type minimal surfaces in $\mathbb{H}^{2} \times \mathbb{R}[\mathrm{Ha}$. Once we have these catenoids, we finish by using the maximum principle similarly to the Euclidean case.

6.3. The classification of entire graphs. In this section we will describe the space of entire graphs of critical $\mathrm{CMC}$ in $\mathbb{E}^{3}(\kappa, \tau)$. Such a description follows from the works of Fernández-Mira [FeMi1, FeMi3], Hauswirth-Rosenberg-Spruck [HRS] and Daniel-Hauswirth DaHa, and is contained in Theorems 6.10 and 6.11. We expose here a unified perspective to this subject. First, we have

Theorem 6.10 ( $\mathrm{DaHa}, \mathrm{FeMi}, \mathrm{HRS})$ ). The following conditions are equivalent for a surface of critical $C M C$ in $\mathbb{E}^{3}(\kappa, \tau)$ :

(1) It is an entire graph.

(2) It is a complete multigraph.

(3) $u^{2} d s^{2}$ is a complete Riemannian metric (where $u$ is the angle function and $d s^{2}$ the metric of the surface).

In particular, the sister correspondence preserves entire graphs of critical CMC.

Let us make some comments on this theorem. First, Hauswirth, Rosenberg and Spruck proved $(2) \Rightarrow(1)$ for $H=1 / 2$ surfaces in $\mathbb{H}^{2} \times \mathbb{R}$. Second, the authors proved in FeMi3] that $(3) \Rightarrow(1)$ (for any surface in $\mathbb{E}^{3}(\kappa, \tau)$, not necessarily CMC), and that $(1) \Rightarrow(3)$ holds for minimal surfaces in $\mathrm{Nil}_{3}$. Finally, Daniel and Hauswirth showed that $(2) \Rightarrow(1)$ holds for minimal surfaces in $\mathrm{Nil}_{3}$. The rest of the cases can be easily obtained from these results and the sister correspondence (this was first observed in $[\mathrm{DHM}]$ ). 
Proof. It is immediate that $(1) \Rightarrow(2)$. Also, by an eigenvalue estimate, the authors proved in FeMi3 that for arbitrary surfaces in $\mathbb{E}^{3}(\kappa, \tau)$ it holds $u^{2} d s^{2} \leq g_{F}$, where $F=\pi \circ \psi$ is the projection onto $\mathcal{M}^{2}(\kappa)$ of $\psi$. Thus, if $u^{2} d s^{2}$ is complete, $F$ is a local diffeomorphism with complete pullback metric, and by standard topological arguments, $F$ is a diffeomorphism, i.e. (3) $\Rightarrow$ (1) holds.

That $(1) \Rightarrow(3)$ holds for minimal surfaces in $\mathrm{Nil}_{3}$ was also proved in FeMi3: let $X=(F, t): \Sigma \rightarrow \mathrm{Nil}_{3}$ be an entire minimal graph. By Theorem 6.7 there is an entire spacelike CMC graph $f=(F, h): \Sigma \rightarrow \mathrm{E}^{3}$, whose induced metric is $d s_{f}^{2}=u^{2} d s^{2}$. Now we can apply a theorem by Cheng and Yau ChYa which says that spacelike entire CMC graphs in $\mathrm{E}^{3}$ have complete induced metric. Hence $u^{2} d s^{2}$ is complete, as wished.

We will now prove that $(2) \Rightarrow(1)$ holds for minimal surfaces in $\mathrm{Nil}_{3}$. Let us observe that once this is done, we can also prove the theorem for surfaces of critical CMC in all the spaces $\mathbb{E}^{3}(\kappa, \tau)$. Indeed, as any simply connected surface of critical CMC is the sister surface of some minimal surface in $\mathrm{Nil}_{3}$, and as the correspondence preserves the metric and the angle function (therefore it preserves conditions (2) and (3) by passing to the universal covering), we can easily translate the theorem for the case of minimal surfaces in $\mathrm{Nil}_{3}$ to the rest of the spaces. It is important here that we proved $(3) \Rightarrow(1)$ in all spaces.

So, we only need to prove $(2) \Rightarrow(1)$ for minimal surfaces in $\mathrm{Nil}_{3}$. This was done by Daniel and Hauswirth DaHa]. For that, they used their half-space theorem in $\mathrm{Nil}_{3}$ (Theorem 6.9) and an adaptation to $\mathrm{Nil}_{3}$ of the previous proof of $(2) \Rightarrow(1)$ for the case of $H=1 / 2$ surfaces in $\mathbb{H}^{2} \times \mathbb{R}$ given by Hauswirth-Rosenberg-Spruck [HRS.

In order to prove $(2) \Rightarrow(1)$ for minimal surfaces in $\mathrm{Nil}_{3}$, we argue by contradiction. Assume that there exists a complete multigraph $\Sigma$ that is not entire. Then there exists an open set $\Sigma_{0} \subset \Sigma$ that is a graph over a disc $D \subsetneq \mathbb{R}^{2}$ of a function $f$, and a point $q \in \partial D$ such that $f$ does not extend to $q$.

Step 1: For any sequence of points $\left\{q_{n}\right\}$ in $D$ converging to $q$, the sequence of normal vectors at the points $p_{n}=\left(q_{n}, f\left(q_{n}\right)\right) \in \Sigma_{0}$ converges to the horizontal vector orthogonal to $\partial D$ at $q$.

Indeed, as the surface is a multigraph, its angle function $u=\left\langle N, E_{3}\right\rangle$, where $N$ denotes the unit normal vector, is a Jacobi function that does not vanish. As a result of this, $\Sigma$ is (strongly) stable, and has bounded geometry. This means that locally around any $p_{n}$ we can write the surface as the graph (in exponential coordinates) over a disc of radius $\delta$ of its tangent plane, where $\delta$ is a universal constant depending only on $\Sigma$. This neighborhood of $p_{n}$ will be denoted by $\mathcal{G}\left(p_{n}\right)$. The limit of the normal vectors $\left\{N\left(p_{n}\right)\right\}$ must be a horizontal vector since otherwise, the piece $\mathcal{G}\left(p_{n}\right)$ of bounded geometry could be extended as a graph beyond $q$, which is impossible. Moreover, the limit vector must be normal to $\partial D$ at $q$ since $\Sigma_{0}$ is a graph over $D$.

Step 2: The function $f$ defining the graph $\Sigma_{0}$ diverges at $q$. Moreover, as we approach $q$, and after translating the surface to the origin, the surfaces converge to a piece of the (translated) vertical plane $P$ passing through $q$ and tangent to $\partial D$.

That $f$ diverges at $q$ is a consequence of the completeness of $\Sigma$, and the last part can be proved by following the ideas of Collin and Rosenberg in CoRo. We will assume that $P$ is the plane $\left\{x_{1}=c\right\}$.

Step 3: $\Sigma$ contains a graph $\mathcal{G}$ over a domain of the form $U_{\epsilon}=(c-\epsilon, c) \times \mathbb{R} \subset \mathbb{R}^{2}$. Moreover, this graph is disjoint from $P$ and asymptotic to it as one approaches $q$.

The graph $\mathcal{G}$ is obtained by analytical continuation of the surfaces $\mathcal{G}\left(p_{n}\right)$ used in the first step, and after a careful study of the behavior of the intersection curves of these graphs and the planes parallel to $P$. 
Finally, the contradiction follows from the half-space theorem (Theorem 6.9). Recall that, although $\mathcal{G}$ has boundary and the theorem is formulated for surfaces without boundary, its proof applies to this case, and so we are done.

Once here, we investigate the Bernstein problem for entire graphs of critical CMC in $\mathbb{E}^{3}(\kappa, \tau)$, i.e. the classification of such entire graphs (recall here that CMC graphs in $\mathbb{E}^{3}(\kappa, \tau)$ satisfy the elliptic PDE (5) $)$. The terminology comes from the classical Bernstein theorem: entire minimal graphs in $\mathbb{R}^{3}$ are planes. Equivalently, any solution to the minimal graph equation

$$
\left(1+f_{y}^{2}\right) f_{x x}-2 f_{x} f_{y} f_{x y}+\left(1+f_{x}^{2}\right) f_{y y}=0
$$

defined on the whole plane is linear.

It is interesting to compare this result with the Bernstein problem in $\mathrm{Nil}_{3}$, i.e. the classification of entire minimal graphs in $\mathrm{Nil}_{3}$. This corresponds to classifying all global solutions to the PDE (6). Observe that taking $\tau=0$ in (6) we obtain the classical equation (13), i.e. the classical case considered by Bernstein appears as a limit of the Heisenberg case.

There exists, however, a great difference between both situations. The following result classifies the entire graphs of critical CMC in $\mathbb{E}^{3}(\kappa, \tau)$, by parametrizing the moduli space of such entire graphs in terms of holomorphic quadratic differentials. It was obtained first for minimal graphs in $\mathrm{Nil}_{3}$ by the authors [FeMi3], and shortly thereafter by Daniel and Hauswirth DaHa] for $H=1 / 2$ graphs in $\mathbb{H}^{2} \times \mathbb{R}$. The general case follows easily from the Heisenberg case and Theorem 6.10 using the sister correspondence (this was observed first in [DHM] $)$.

Theorem 6.11 (Fernández-Mira, Daniel-Hauswirth). Let $Q d z^{2}$ denote a holomorphic quadratic differential on $\Sigma \equiv \mathbb{C}$ or $\mathbb{D}$, such that $Q \not \equiv 0$ if $\Sigma \equiv \mathbb{C}$, and let $H^{2}=-\kappa / 4$.

There exists a 2-parameter family of entire CMCH graphs in $\mathbb{E}^{3}(\kappa, \tau)$ whose AbreschRosenberg differential agrees with $Q d z^{2}$. These graphs are generically non-congruent.

And conversely, these are all the entire graphs of critical $C M C$ in $\mathbb{E}^{3}(\kappa, \tau)$.

At this point, the proof for the case of minimal surfaces in $\mathrm{Nil}_{3}$ is a consequence of Theorem 6.7 and the following result by Wan and Wan-Au Wan, WaAu on spacelike entire CMC graphs in $\mathrm{E}^{3}$ : for any holomorphic quadratic differential as above, there exists a unique (up to isometries) spacelike entire CMC $1 / 2$ graph in $E^{3}$ with Hopf differential $Q d z^{2}$. The 2-parameter family of non-congruent graphs in $\mathbb{E}^{3}(\kappa, \tau)$ comes from the loss of ambient isometries (from 6 dimensions to 4 dimensions) when passing from $\mathrm{E}^{3}$ to $\mathrm{Nil}_{3}$.

The remaining cases of critical CMC graphs follow since by Theorem 6.10 the sister correspondence preserves entire graphs.

6.4. Open Problems. As explained in Section 3, entire graphs are stable. It is conjectured that entire graphs and vertical cylinders are the only stable critical CMC surfaces (this has been proved for parabolic conformal type in [MaPR]). Related to this is the question of non-existence of complete stable $H>1 / 2$ surfaces in $\mathbb{H}^{2} \times \mathbb{R}$ (proved for $H>1 / \sqrt{3}$ by Nelli-Rosenberg, NeRo2 ).

Also, not much is known about properly embedded surfaces of critical CMC and non-trivial topology. Can one obtain them by conjugate Plateau constructions, or by integrable systems techniques? Another remarkable problem is to establish the strong half-space theorem in $\mathrm{Nil}_{3}$ : are two disjoint properly embedded minimal surfaces in $\mathrm{Nil}_{3}$ necessarily two parallel vertical planes, or two parallel entire minimal graphs? 


\section{Minimal surfaces in $\mathbb{H}^{2} \times \mathbb{R}$ and $\mathbb{S}^{2} \times \mathbb{R}$}

Minimal surfaces in product spaces admit a special treatment, due to several reasons. One of them is the following: if $\psi=(N, h): \Sigma \rightarrow M^{2} \times \mathbb{R}$ is a minimal surface immersed in the product space $M^{2} \times \mathbb{R}$, where $\left(M^{2}, g\right)$ is a Riemannian surface, then the horizontal projection $N: \Sigma \rightarrow M^{2}$ is a harmonic map and the height function $h: \Sigma \rightarrow \mathbb{R}$ is a harmonic function. This implies, for instance, that compact minimal surfaces in $M^{2} \times \mathbb{R}$ only exist if $M^{2}$ is compact (in particular, if $M^{2}=\mathbb{S}^{2}$ ), and the only ones are the slices $M^{2} \times\left\{t_{0}\right\}$.

Another important fact about minimal surfaces in $M^{2} \times \mathbb{R}$ is that there is a natural notion of minimal graph over a domain $\Omega \subset M^{2}$, and that this graph satisfies a simple elliptic PDE in divergence form. This fact together with general existence results for solutions to the Plateau problem in Riemannian 3-manifolds allows a good control on the geometry of the surface. Some of the most interesting results of the theory of minimal surfaces in product spaces come from the interplay between the information provided by harmonic maps and by Plateau constructions and the minimal graph equation.

Starting with the pioneer work of H. Rosenberg Ros, and W.H Meeks and H. Rosenberg MeRo1, MeRo2], the theory of minimal surfaces in $M^{2} \times \mathbb{R}$ has developed substantially in the last decade. We will only talk here about a few results of special relevance to the theory, and not mention many other important results.

7.1. The Collin-Rosenberg theorem. The classical Bernstein theorem in $\mathbb{R}^{3}$ states that planes are the only entire minimal graphs in $\mathbb{R}^{3}$. This theorem can be extended to the case of product spaces: any entire minimal graph in $M^{2} \times \mathbb{R}$, where $\left(M^{2}, g\right)$ is a complete surface of non-negative curvature, is totally geodesic.

In contrast, in the product space $\mathbb{H}^{2} \times \mathbb{R}$ there is a wide variety of entire minimal graphs. For instance, in NeRo1 Nelli and Rosenberg solved the Dirichlet problem at infinity for the minimal graph equation in $\mathbb{H}^{2} \times \mathbb{R}$. They proved that any Jordan curve at the ideal boundary $\mathbb{S}^{1} \times \mathbb{R} \equiv \partial_{\infty} \mathbb{H}^{2} \times \mathbb{R}$ of $\mathbb{H}^{2} \times \mathbb{R}$ which is a graph over $\mathbb{S}^{1} \equiv \partial_{\infty} \mathbb{H}^{2}$ is the asymptotic boundary of a unique entire minimal graph in $\mathbb{H}^{2} \times \mathbb{R}$ (see GaRo for a proof of this in the more general case of entire minimal graphs in $M^{2} \times \mathbb{R}$, where $\left(M^{2}, g\right)$ is complete, simply connected and with $\left.K_{M} \leq c<0\right)$.

All these entire minimal graphs are hyperbolic, that is, they have the conformal type of the unit disk. The problem of existence of entire minimal graphs of parabolic type (i.e. with the conformal type of $\mathbb{C}$ ) is much harder, and was solved recently by Collin and Rosenberg CoRo.

Theorem 7.1 (Collin-Rosenberg). There exist entire minimal graphs in $\mathbb{H}^{2} \times \mathbb{R}$ of parabolic conformal type.

As the projection onto $\mathbb{H}^{2}$ of a minimal graph is a harmonic diffeomorphism, the above theorem has the following consequence, which solves a major problem in the theory of harmonic maps and disproves a conjecture by R. Schoen and S.T. Yau.

Corollary 7.2 (Collin-Rosenberg). There exist harmonic diffeomorphisms from $\mathbb{C}$ onto $\mathbb{H}^{2}$.

The proof by Collin and Rosenberg is a good example of the interaction between the harmonicity properties of the minimal immersion and the use of Plateau constructions and the minimal graph equation. 
The main idea in the proof is to construct first (non-entire) minimal graphs in $\mathbb{H}^{2} \times \mathbb{R}$ of Scherk type over ideal geodesic polygons, having alternating asymptotic values $+\infty$ and $-\infty$ on the sides of the polygon. This generalizes a classical construction by Jenkins and Serrin in the case of minimal graphs over bounded domains in $\mathbb{R}^{3}$. This construction is done as follows:

Let $\Gamma$ be an ideal polygon of $\mathbb{H}^{2}$, so that all the vertices of $\Gamma$ are at the ideal boundary of $\mathbb{H}^{2}$ and $\Gamma$ has an even number of sides $A_{1}, B_{1}, A_{2}, B_{2} \ldots, A_{k}, B_{k}$, ordered clockwise. At each vertex $a_{i}$, we consider a small enough horocycle $H_{i}$ with $H_{i} \cap H_{j}=\emptyset$. Each $A_{i}$ (resp. $B_{i}$ ) meets exactly two horocycles. Denote by $\widetilde{A}_{i}$ (resp. $\widetilde{B}_{i}$ ), the compact arc of $A_{i}\left(\operatorname{resp} B_{i}\right)$ which is the part of $A_{i}$ outside the two horodisks. We denote by $\left|A_{i}\right|$ the length of $\left|\widetilde{A}_{i}\right|$. Define $\widetilde{B}_{i}$ and $\left|B_{i}\right|$ in the same way.

Now we can consider $a(\Gamma)=\sum_{i=1}^{k}\left|A_{i}\right|$ and $b(\Gamma)=\sum_{i=1}^{k}\left|B_{i}\right|$. We observe that $a(\Gamma)-b(\Gamma)$ does not depend on the choice of the horocycle $H_{i}$ at $a_{i}$, since horocycles with the same point at infinity are equidistant. Keeping in mind these data, we can state the following theorem by Collin-Rosenberg [CoRo] (see also Nelli-Rosenberg [NeRo1]):

Theorem 7.3. ([NeRo1], [CoRo]) There is a (unique up to additive constants) solution to the minimal surface equation in the polygonal domain $P$, equal to $+\infty$ on $A_{i}$ and $-\infty$ on $B_{i}$, if and only if the following conditions are satisfied:

1. $a(\Gamma)=b(\Gamma)$,

2. For each inscribed polygon $\mathcal{P}$ in $\Gamma, \mathcal{P} \neq \Gamma$, and for some choice of horocycles at the vertices, one has

$$
2 a(\mathcal{P})<|\mathcal{P}| \text { and } 2 b(\mathcal{P})<|\mathcal{P}|
$$

All these examples have the conformal type of $\mathbb{C}$. Once there, Collin and Rosenberg designed a way of enlarging a given Scherk-type graph over the interior of some $\Gamma \subset \mathbb{H}^{2}$ into another one with more sides, and so that: (1) the extended surface is $C^{2}$-close to the original one over an arbitrary compact set in the interior of $\Gamma$, and (2) there is a control on the conformal radius on adequate compact annuli on the surface.

By passing to the limit in this sequence of minimal graphs over larger and larger domains, they obtained an entire minimal graph in $\mathbb{H}^{2} \times \mathbb{R}$ which, by the control on the conformal radii of these annuli, has the conformal type of $\mathbb{C}$.

Remark 7.4. The Collin-Rosenberg theorem has been extended by J.A. Gálvez and H. Rosenberg [GaRo] to more general product spaces $M^{2} \times \mathbb{R}$ : there exist entire minimal graphs of parabolic conformal type on $M^{2} \times \mathbb{R}$, where $\left(M^{2}, g\right)$ is any complete simply connected Riemannian surface with Gaussian curvature $K_{M} \leq c<0$ ( $K_{M}$ not constant).

7.2. Minimal surfaces of finite total curvature in $\mathbb{H}^{2} \times \mathbb{R}$. One of the most studied families among minimal surfaces in $\mathbb{R}^{3}$ are the complete minimal surfaces of finite total curvature (FTC for short). A minimal surface $\Sigma$ is said to have FTC if its Gaussian curvature $K$ satisfies

$$
\left|\int_{\Sigma} K d A\right|<\infty .
$$

By classical theorems of Huber and Osserman, complete FTC minimal surfaces in $\mathbb{R}^{3}$ are conformally equivalent to a compact Riemann surface minus a finite number of points. Moreover, the Gauss map extends meromorphically to the punctures, and the total curvature of the surface is a multiple of $-4 \pi$. A key point here is that the Gauss map of a minimal surface in $\mathbb{R}^{3}$ is conformal. 
In $\mathbb{H}^{2} \times \mathbb{R}$ there is no conformal Gauss map for minimal surfaces. Nonetheless, using the global theory of harmonic maps into $\mathbb{H}^{2}$, L. Hauswirth and H. Rosenberg HaRo were able to prove that a similar situation holds in $\mathbb{H}^{2} \times \mathbb{R}$.

Theorem 7.5 (Hauswirth-Rosenberg). Let $X$ be a complete minimal immersion of $\Sigma$ in $\mathbb{H}^{2} \times \mathbb{R}$ with finite total curvature. Then

1. $\Sigma$ is conformally equivalent to a Riemann surface punctured at a finite number of points, $\Sigma \equiv \bar{M}_{g}-\left\{p_{1} \ldots, p_{k}\right\}$.

2. $Q d z^{2}:=h_{z}^{2} d z^{2}$ is holomorphic on $M$ and extends meromorphically to each puncture. If we parameterize each puncture $p_{i}$ by the exterior of a disk of radius $r$, and if $Q(z) d z^{2}=z^{2 m_{i}}(d z)^{2}$ at $p_{i}$, then $m_{i} \geqslant-1$.

3. The third coordinate $u$ of the unit normal tends to zero uniformly at each puncture.

4. The total curvature is a multiple of $2 \pi$ :

$$
\int_{\Sigma} K d A=2 \pi\left(2-2 g-2 k-\sum_{i=1}^{k} m_{i}\right) .
$$

As a consequence, every end of a finite total curvature surface is uniformly asymptotic to a Scherk type graph described in Theorem 7.3 .

Proof. The first step is to prove that locally around an end, $Q d z^{2}$ only has at most a finite number of zeroes. Then a Huber theorem and an argument of Osserman give that the ends are conformally a punctured disk, and $Q d z^{2}$ extends meromorphically to the puncture. The final part of the behavior of $Q d z^{2}$ follows from the fact that $Q d z^{2}=h_{z}^{2} d z^{2}$, where $h$ is the height function of the surface.

To prove that $u$ goes to 0 at the ends, take an annular neighborhood of an end where $Q d z^{2}$ does not vanish. Then reparameterize this annulus by $w=\int \sqrt{Q} d z$. The metric conformal factor in these coordinates satisfies a sinh-Gordon equation, and the Gaussian curvature monotonically decreases to zero. Then, estimates on the growth of solutions of the sinh-Gordon equation allows one to conclude that, at a finite total curvature end, the tangent plane becomes vertical and the metric becomes flat.

Finally, the expression for the total curvature follows from Gauss-Bonnet formula and the estimates for the sinh-Gordon equation obtained before.

In $\mathrm{HaRO}$, the following question was also raised: are there complete non simply connected minimal surfaces with FTC in $\mathbb{H}^{2} \times \mathbb{R}$ ? Notice that rotational catenoids have infinite total curvature. Actually, at that time, the only known complete FTC minimal surfaces were the Scherk type graphs.

This question was positively answered by J. Pyo Pyo and also, independently, by Rodríguez and Morabito RoMo. Pyo constructed a 1-parameter family of genus zero properly embedded minimal surfaces in $\mathbb{H}^{2} \times \mathbb{R}$ with $k$ ends for $k \geq 2$, similar to the $k$-noids in $\mathbb{R}^{3}$ (although the first ones are embedded and the $k$-noids in $\mathbb{R}^{3}$ are not). They have total curvature $4 \pi(1-k)$, and are asymptotic to vertical planes at infinity. These surfaces are obtained as the conjugate surfaces of minimal graphs over infinite geodesic triangles in $\mathbb{H}^{2}$ that are asymptotic to vertical planes at infinity.

Very shortly thereafter, M. Rodríguez and F. Morabito discovered independently a larger family of FTC minimal surfaces, containing the previous ones. It is a $(2 k-$ 2)-parameter of properly embedded FTC minimal surfaces of genus zero with $k$ ends, obtained as the limits of simply periodic minimal surfaces called saddle towers, that are 
invariant by a vertical translation of vector $(0,0,2 l)$. Taking limits when $l \rightarrow \infty$, they obtain genus zero minimal surfaces with $k$ ends and total curvature $4 \pi(1-k)$ that are symmetric with respect to the reflection over the slice $\mathbb{H}^{2} \times\{0\}$. The surfaces found by Pyo appear when the ends are placed in symmetric positions.

7.3. Open problems. In $\mathrm{Ha}$, L. Hauswirth constructed a family of Riemann type minimal surfaces in $\mathbb{H}^{2} \times \mathbb{R}$ and $\mathbb{S}^{2} \times \mathbb{R}$, characterized by the property of being foliated by curves of constant curvature. It is a conjecture by W. Meeks and H. Rosenberg that in $\mathbb{S}^{2} \times \mathbb{R}$ they are the only properly embedded minimal annuli. An approach for solving this conjecture using integrable systems techniques has been recently developed by L. Hauswirth and M. Schmidt. Another natural problem is to obtain classification results for properly embedded minimal surfaces of finite total curvature and a given simple topology in $\mathbb{R}^{3}$.

Schoen and Yau proved there is no harmonic diffeomorphism from the disk to a complete surface of non-negative curvature. Can there be such a harmonic diffeomorphism onto a complete parabolic surface? This is a question by J.A. Gálvez.

\section{References}

[AbRo1] U. Abresch, H. Rosenberg, A Hopf differential for constant mean curvature surfaces in $\mathbb{S}^{2} \times \mathbb{R}$ and $\mathbb{H}^{2} \times \mathbb{R}$, Acta Math. 193 (2004), 141-174.

[AbRo2] U. Abresch, H. Rosenberg, Generalized Hopf differentials, Mat. Contemp. 28 (2005), 1-28.

[AEG1] J.A. Aledo, J.M.Espinar, J.A. Gálvez, Height estimates for surfaces with positive mean curvature in $\mathbb{M} \times \mathbb{R}$. Illinois Journal of Math., 52 (2008), 203-211.

[Bon] F. Bonahon, Geometric structures on 3-manifolds. In Handbook of Geometric Topology, pages 93-164. North-Holland, Amsterdam, 2002.

[ChYa] S.Y. Cheng, S.T. Yau, Maximal spacelike hypersurfaces in the Lorentz-Minkowski spaces, Ann. of Math. 104 (1976), 407-419.

[CoRo] P. Collin, H. Rosenberg, Construction of harmonic diffeomorphisms and minimal graphs, Ann. of Math., to appear (2007).

[Dan1] B. Daniel, Isometric immersions into 3-dimensional homogeneous manifolds, Comment. Math. Helv. 82 (2007), 87-131.

[Dan2] B. Daniel, The Gauss map of minimal surfaces in the Heisenberg group, preprint, 2006, arXiv:math/0606299

[DFM] B. Daniel, I. Fernández, P. Mira, Surfaces of critical constant mean curvature. Work in progress.

[DaHa] B. Daniel, L. Hauswirth, Half-space theorem, embedded minimal annuli and minimal graphs in the Heisenberg group. Proc. Lond. Math. Soc. (3), 98 no.2 (2009), 445-470.

[DHM] B. Daniel, L. Hauswirth, P. Mira, Constant mean curvature surfaces in homogeneous manifolds, preprint, 2009. Published preliminarly by the Korea Institute for Advanced Study.

[DaMi] B. Daniel, P. Mira, Existence and uniqueness of constant mean curvature spheres in $\mathrm{Sol}_{3}$. Preprint, 2008, arXiv:0812.3059 
[dCF] M.P. do Carmo, I. Fernández, Rotationally invariant CMC disks in product space, Forum Math. 21 (2009), 951-963.

[EGR] J.M. Espinar, J.A. Gálvez, H. Rosenberg, Complete surfaces with positive extrinsic curvature in product spaces, Comment. Math. Helv., 84 (2009), 351-386.

[EsRo] J.M. Espinar, H. Rosenberg, Complete constant mean curvature surfaces in homogeneous spaces, Comment. Math. Helv., to appear (2009).

[FeMi1] I. Fernández, P. Mira, Harmonic maps and constant mean curvature surfaces in $\mathbb{H}^{2} \times \mathbb{R}$, Amer. J. Math. 129 (2007), 1145-1181.

[FeMi2] I. Fernández, P. Mira, A characterization of constant mean curvature surfaces in homogeneous 3-manifolds, Diff. Geom. Appl., 25 (2007), 281-289.

[FeMi3] I. Fernández, P. Mira, Holomorphic quadratic differentials and the Bernstein problem in Heisenberg space. Trans. Amer. Math. Soc., 361, no 11, (2009), 57375752.

[FoWo] A.P. Fordy, J.C. Wood. Harmonic maps and integrable systems. Aspects of Mathematics, vol. E23, by Vieweg, Braunschweig/Wiesbaden, 1994.

[GMM] J.A. Gálvez, A. Martínez, P. Mira, The Bonnet problem for surfaces in homogeneous 3-manifolds, Comm. Anal. Geom. 16 (2008), 907-935.

[GaRo] J.A. Gálvez, H. Rosenberg, Minimal surfaces and harmonic diffeomorphisms from the complex plane onto a Hadamard surface. Preprint, 2008, arXiv:0807.0997.

[Ha] L. Hauswirth, Minimal surfaces of Riemann type in three dimensional product manifolds. Pacific J. Math., 224, no.1 (2006), 91-117.

[HaRo] L. Hauswrith, H. Rosenberg. Minimal surfaces of finite total curvature in $\mathbb{H} \times \mathbb{R}$. Mat. Contemp. 31 (2006), 65-80.

[HRS] L. Hauswirth, H. Rosenberg, J. Spruck. On complete mean curvature $\frac{1}{2}$ surfaces in $\mathbb{H}^{2} \times \mathbb{R}$. Comm. Anal. Geom., 16, no.5 (2008), 989-1005.

[HoMe] D. Hoffman, W. H. Meeks III. The strong halfspace theorem for minimal surfaces. Invent. Math. 101, no.2 (1990), 373-377.

[HsHs] W.Y. Hsiang, W.T. Hsiang, On the uniqueness of isoperimetric solutions and imbedded soap bubbles in noncompact symmetric spaces I, Invent. Math. 98 (1989), $39-58$.

[Lee] H. Lee. Extension of the duality between minimal surfaces and maximal surfaces. Preprint, 2009.

[MaPR] M. Manzano, J. Pérez, M. Rodríguez. Parabolic stable surfaces with constant mean curvature. Preprint, 2009, arXiv:0910.5373.

[MPR] W. H. Meeks III, J. Pérez, A. Ros. Stable constant mean curvature surfaces, Handbook of Geometric Analysis no.1 (2008).

[Mee] W.H. Meeks. Constant mean curvature surfaces in homogeneous 3-manifolds. Preprint (2009).

[MeRo1] W.H. Meeks, H. Rosenberg, The theory of minimal surfaces in $M \times \mathbb{R}$, Comment. Math. Helv. 80 (2005), 811-858.

[MeRo2] W.H. Meeks, H. Rosenberg, Stable minimal surfaces in $M \times \mathbb{R}$, J. Differential Geom. 68 (2004), 515-534. 
[MoUr] S. Montiel, F. Urbano, A Willmore functional for compact surfaces in the complex projective plane, J. Reine Angew. Math. 546 (2002), 139-154.

[NeRo1] B. Nelli, H. Rosenberg. Minimal surfaces in $\mathbb{H}^{2} \times \mathbb{R}$. Bull. Braz. Math. Soc., 33, no.2 (2002), 263-292.

[NeRo2] B. Nelli, H. Rosenberg. Global properties of constant mean curvature surfaces in $\mathbb{H}^{2} \times \mathbb{R}$. Pacific J. Math. 226, no-1 (2006), 137-152.

[Oss] R. Osserman. A survey on minimal surfaces. Dover Publications Inc., New York, second edition, 1986.

[Ped] R. Pedrosa. The isoperimetric problem in spherical cylinders, Ann. Global Anal. Geom. 26 (2004), 333-354.

[Pyo] J. Pyo. New examples of minimal surfaces in $\mathbb{H}^{2} \times \mathbb{R}$. Preprint, 2009, arXiv:0911.5577.

[RoMo] M. Rodríguez, F. Morabito. Saddle towers in $\mathbb{H}^{2} \times \mathbb{R}$. Preprint, 2009, arXiv:0910.5676.

[Ros] H. Rosenberg, Minimal surfaces in $M^{2} \times \mathbb{R}$, Illinois J. Math. 46 (2002), 1177-1195.

[SaE] R. Sa Earp, Parabolic and hyperbolic screw motion surfaces in $\mathbb{H}^{2} \times \mathbb{R}$, J. Austr. Math. Soc., 85 (2008), 113-143.

[SaTo] R. Sa Earp, E. Toubiana, Screw motion surfaces in $\mathbb{H}^{2} \times \mathbb{R}$ and $\mathbb{S}^{2} \times \mathbb{R}$, Illinois J. Math. 49 (2005), 1323-1362.

[Sco] P. Scott. The geometries of 3-manifolds, Bull. London Math. Soc. 15 (1983), 401487.

[Tor] F. Torralbo. Rotationally invariant constant mean curvature surfaces in homogeneous 3-manifolds. Diff. Geom. Appl., to appear (2009), arXiv:0911.5128.

[ToUr] F. Torralbo, F. Urbano. Compact stable constant mean curvature surfaces in the Berger 3-spheres. Preprint, 2009, arXiv:0906.1439.

[Wan] T.Y. Wan, Constant mean curvature surface harmonic map and universal Teichmuller space, J. Differential Geom. 35 (1992), 643-657.

[WaAu] T.Y. Wan, T.K. Au, Parabolic constant mean curvature spacelike surfaces, Proc. Amer. Math. Soc. 120 (1994), 559-564.

The authors were partially supported by MEC-FEDER, Grant No. MTM2007-65249, Junta de Andalucía Grant No. FQM325 and the Programme in Support of Excellence Groups of Murcia, by Fundación Séneca, R.A.S.T 2007-2010, reference 04540/GERM/06 and Junta de Andalucía, reference P06-FQM-01642."

Isabel Fernández, Universidad de Sevilla (Spain)

E-mail: isafer@us.es

Pablo Mira, Universidad Politécnica de Cartagena (Spain)

E-mail: pablo.mira@upct.es 